\title{
A Differential Pilot Protection Scheme for MMC Based DC Grid Resilient to Communication Failure
}

\author{
Wang Xiang, Member, IEEE, Haobo Zhang, Saizhao Yang, Meng Zhou, Weixing Lin, Member, IEEE, \\ Jinyu Wen, Member, IEEE
}

\begin{abstract}
Primary single-end line protection strategies of MMC HVDC systems are difficult to make a tradeoff between fast detection speed and high reliability. To improve reliability, the pilot protection schemes based on communication and data exchange can be adopted. However, the communication-based schemes suffer from potential communication failure problems, such as data error, data loss and time synchronization error. To avoid blocking of protection devices during communication failures, a resilience-oriented differential pilot protection method is proposed in this paper. To address the problem of synchronization error, a startup element based on the multiresolution morphological gradient (MMG) of traveling wave is proposed. For the problems of data error and data loss in communication, the sampled data are preprocessed by the morphological filtering $(M F)$. And the correlation of traveling waves is used to identify the internal and external faults; the ratio of the morphological gradient of pole voltages is adopted to discriminate the faulty poles. A four-terminal MMC based DC grid model is built in PSCAD/EMTDC interfaced with the optical fiberbased communication system built in MATLAB/Simulink. The simulation results show that the protection scheme can effectively identify the faults against serious communication problems of $1 \%$ bit error rate and $5 \%$ data loss.
\end{abstract}

Index Terms- DC grid; data communication; differential protection; MMC; morphological gradient; morphological filtering.

\section{INTRODUCTION}

Modular multilevel converter (MMC) based DC grid is recognized as a potential candidate to integrate large-scale renewable energy [1][2]. When DC faults happen, the fault current will increase rapidly due to the low inertia of the DC system and the infeed of multiple converters [3][4]. To protect the power electronic devices, ultra-fast DC fault protection is required to identify the fault lines within $2 \sim 3 \mathrm{~ms}$ [5]. Many protection schemes in time-domain and frequency-domain are proposed, utilizing the transient voltage and current traveling waves (TWs) and the boundary protection algorithms based on current-limiting inductors [6][7]. Although these protection schemes are quite fast, they suffer from low reliability under

This work is sponsored by the Joint Funds of the National Natural Science Foundation of China (U1766211) and the National Natural Science Foundation of China (52007075) and the Xinjiang Autonomous Region Key R\&D Projects (2020B02002). (Corresponding author: Meng Zhou)

W. Xiang is with the Department of Electronic and Electrical Engineering, University of Strathclyde, Glasgow, G1 1XW, U.K. (e-mail: xiangwang1003@foxmail.com).

H. Zhang, S. Yang, M. Zhou, J. Wen are with the State Key Laboratory of Advanced Electromagnetic Engineering and Technology, Huazhong University of Science and Technology, Wuhan 430074, China. (e-mail zhanghaobo9902@foxmail.com, saizhaoyang@foxmail.com, zhoumeng $4077 @$ foxmail.com, jinyu.wen@hust.edu.cn).

W. Lin is with the TBEA SunOasis Co,. Ltd, Urumuchi 830011, Xinjiang, China. (e-mail: weixinglin@foxmail.com). remote and high-resistance faults. To overcome the disadvantages, the pilot protection schemes are proposed [8].

The pilot protection depends on the optical fiber cables to communicate between converter stations, which are relatively reliable and fast. However, the optical cables still exist some communication failure problems, i.e., the failure of communication equipment interface, dispersion and interference of optical signals [9]. In practice, converter stations adopting the pilot protection are equipped with communication fault detection devices. Once the communication failure occurs without redundant communication lines, to prevent the protection from malfunction, the detection device will be blocked and re-opened after a pre-determined delay [10]. This blocking of protection will delay the detection time and even result in the refusal-operation of protection.

To reduce the dependency on communication, some researchers employ the directional pilot protection that transmits only logical signals. But when communication failures occur, the receiving terminals are still unable to process and recover the affected logic signals. Thus, the protection devices will also be blocked. Moreover, the directional protection schemes use the single-end measurements, which need high sampling frequency and are inefficient to detect faults with high resistances.

Compared with the directional pilot protection, the differential pilot protection transmits the electrical quantities between converter stations, of which the affected data can be processed and recovered, providing a suitable solution to improve the resilience to communication failures. But the impact of communication failures on the performance of the protection schemes is rarely mentioned. References [11] and [12] use the similarity principle of traveling waves for fault identification. Reference [13] calculates the DC power difference between the positive pole at one terminal and the negative pole at the other terminal to detect the faults. They perform good effectiveness under high-resistance faults and synchronization errors, but the performance under other communication failures needs to be further discussed and explored.

To address the above challenge, this paper proposes a novel differential pilot protection resilient to communication failures. Firstly, the common communication failure problems and their impact on the DC fault protection algorithm are summarized in Section II. Then, the analysis of fault traveling wave characteristics of a four-terminal MMC based HVDC grid is presented in Section III. The morphological gradient (MG) of the traveling wave is employed as the start-up element to accurately determine the zero time of the data time-stamp in 
This paper is a post-print of a paper submitted to and accepted for publication in IEEE Journal of Emerging and Selected Topics in Power Electronics and is subject to Institution of Electrical and Electronic Engineering Copyright. The copy of record is available at IEEE Xplore Digital Library

stations, which overcomes the problem caused by clock time error between both stations. The correlation of voltage traveling waves pre-processed by morphological filtering is used as the criterion for fault identification, avoiding the impact of bit errors and data loss. The overall protection algorithm is elaborated in Section IV. And the simulation validations including the communication system and the electromagnetic HVDC grid are carried out in Section V. The robustness analysis against communication failure problems is conducted in Section VI. Finally, the conclusion is drawn in Section VII.

\section{Communication Failure Problems in Pilot PROTECTION}

In some countries like China, the communication channels for transmission line protection with voltage levels no smaller than $220 \mathrm{kV}$ mostly use optical fiber cables. Thus, optical fiber cable-based communication is studied in this paper.

\section{a. Synchronization error problem}

The communication system between two stations needs to be synchronized to coordinate the protection algorithm. According to the standards of some countries like China, USA, South Korea, external clock synchronization such as the GPS synchronization scheme is not adopted for relay protection devices. Thus, only the point-to-point ping-pong synchronization scheme can be adopted [14]-[16]. However, in the case of asymmetrical channel delay, the channel transmission delay cannot be calculated correctly [16]. Thus, there will be a timing error between the two stations, which causes the sampling time difference and out of synchronization [17][18]. This synchronization error mainly affects the current differential protection. It will cause protection malfunction during the change of operating states, leading to an increase of protective thresholds and deterioration of the protection sensitivity. To overcome the issue caused by asymmetrical channel delay, some solutions like fallback algorithms from Schweitzer Engineering Labs are proposed in [16]. However, it needs the change of operation modes.

\section{b. Data error problem}

Optical fiber cables usually use a composite overhead ground wire, which is exposed to air and vulnerable to external physical damage and chemical corrosion. Under long-term operation, data errors and even communication interruptions may occur in communication systems [9]. To evaluate the severity of data errors, the bit error rate (BER) is used in engineering. After detecting the error bits, the differential protection needs to be re-started. Thus, data errors mainly prolong the protection action time.

\section{c. Data Loss problem}

As affected by external disturbance, the long-distance optical fiber cables increase the transmission loss, severe attenuation in signals, which eventually cause data loss for long-distance communication. The data loss will not only prolong the detection delay, but also cause protection malfunction. If there are no redundant communication lines, when a large amount of data loss happens, the protection device needs to be blocked for a long time and the communication equipment needs maintenance as well as the cables.

\section{TRaVEling WaVe ANALYSIS OF MMC HVDC GRID UNDER DC LINE FAULTS}

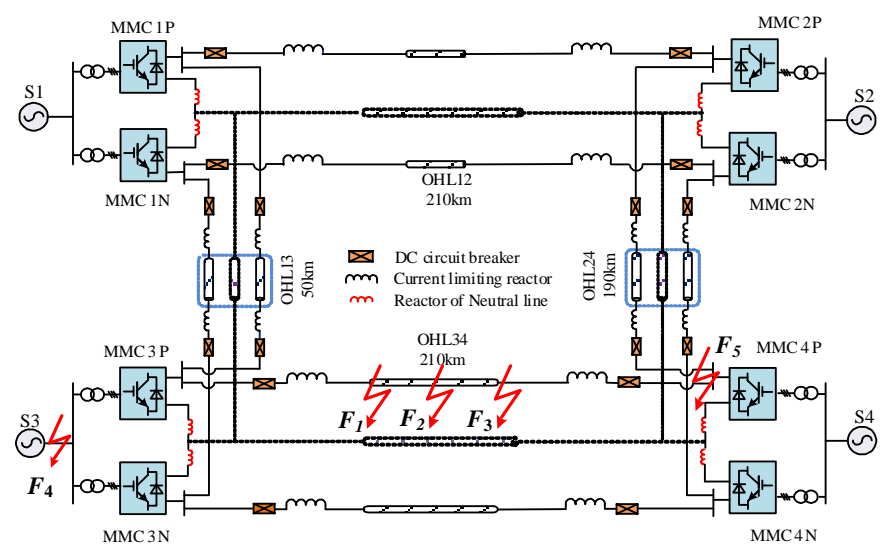

Fig. 1 Topology of a four-terminal meshed DC grid.

Fig. 1 shows a typical four-terminal meshed bipolar DC grid topology. The four terminals MMC1-MMC4 adopt the halfbridge sub-module (HB-SM) based modular multilevel converter (MMC) technology. The high power hybrid DC circuit breakers associated with protection devices are installed at the terminals of each overhead line (OHL). To limit the fault current rise rate, current limiting inductors are implemented at the polar and ground lines.

\section{A. Internal DC Line Faults}

Supposing a pole-to-pole (PTP) DC short-circuit fault occurs at OHL 34, the equivalent circuit is shown in Fig. 2. Where $V_{F I}$ represents the imposed fault voltage source at the fault point. Denoting the protection devices on OHL 34 at the MMC3 and MMC4 sides are respective $M$ and $N$. Taking the relay $M$ as an example, $I_{M F_{-} p}$ and $V_{M F_{-} p}\left(I_{M F_{-} n}\right.$ and $\left.V_{M F_{-} n}\right)$ respectively represent the measured fault current and voltage of the positive (negative) pole line at $M$.

In the bipolar MMC-HVDC systems, to decouple the couplings between positive and negative transmission lines, phase-modal transformation is employed. The line-mode voltage $V_{M F I}(\mathrm{t})$ and the line-mode current $I_{M F I}(\mathrm{t})$ at relay $M$ can be obtained:

$$
\begin{aligned}
& {\left[\begin{array}{l}
V_{M F 1} \\
V_{M F 0}
\end{array}\right]=\boldsymbol{S} V_{F}(t)=\boldsymbol{S}\left[\begin{array}{l}
V_{M F_{-} p} \\
V_{M F_{-} n}
\end{array}\right]} \\
& {\left[\begin{array}{c}
I_{M F 1} \\
I_{M F 0}
\end{array}\right]=\boldsymbol{S} \boldsymbol{I}_{F}(t)=\boldsymbol{S}\left[\begin{array}{l}
I_{M F_{-} p} \\
I_{M F_{-} n}
\end{array}\right]}
\end{aligned}
$$

where $S$ is the phase-modal transformation matrix, $S=\left[\begin{array}{cc}1 & -1 \\ 1 & 1\end{array}\right]$.

Define the traveling wave propagating from the bus bar to the line as the forward traveling wave. Otherwise, it is the backward traveling wave. The forward traveling wave $V_{M F I f}$ and the backward traveling wave $V_{M F I} b$ of the line-mode voltage can be expressed as: 
This paper is a post-print of a paper submitted to and accepted for publication in IEEE Journal of Emerging and Selected Topics in Power Electronics and is subject to Institution of Electrical and Electronic Engineering Copyright. The copy of record is available at IEEE Xplore Digital Library

$$
\left\{\begin{array}{l}
V_{M F 1_{-} f}=\frac{V_{M F 1}(t)+Z_{1} I_{M F 1}(t)}{2} \\
V_{M F 1_{-} b}=\frac{V_{M F 1}(t)-Z_{1} I_{M F 1}(t)}{2}
\end{array}\right.
$$

where $Z_{l}$ is the line-mode component of the wave impedance $Z_{C}$. Similarly, the forward traveling wave $V_{N F l_{f} f}$ and the backward traveling wave $V_{N F I_{-} b}$ of the line-mode fault voltages at relay $N$ can be obtained.

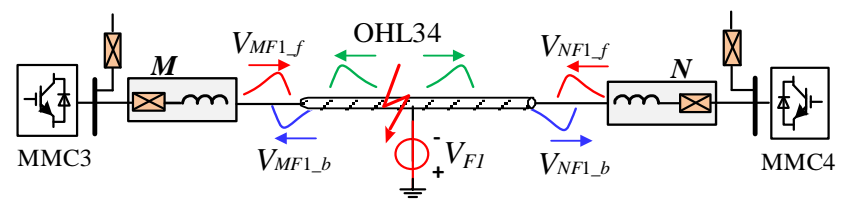

Fig. 2 Equivalent circuit under OHL 34 DC fault.

The voltage traveling wave $V_{F 1}(t)$ induced by the fault will propagate towards $M$ and $N$ and be refracted and reflected at both ends of the line and the fault point. Fig. 3 shows the multiple refraction and reflection process of the fault voltage traveling waves. In Fig. $3, \beta_{M}, \beta_{N}$, and $\beta_{f}$ are respectively the reflection coefficients of the traveling wave at relays $M, N$ and the fault point. $\alpha_{f}$ is the traveling wave refraction coefficient at the fault point.

The forward line-mode voltage traveling wave $V_{M F 1_{f} f}$ at $M$ and the backward line-mode voltage traveling wave $V_{N F 1 \_b}$ at $N$ can be calculated as shown in Table 1. According to the parameters listed in Section $\mathrm{V}$, the $V_{M F 1_{-} f}$ and $V_{N F 1_{-} b}$ are measured as depicted in Fig. 4. From Table 1 and Fig. 4, it can be seen that with the increase of fault time, the two waveforms perform weak similarity after multiple refraction and reflection.

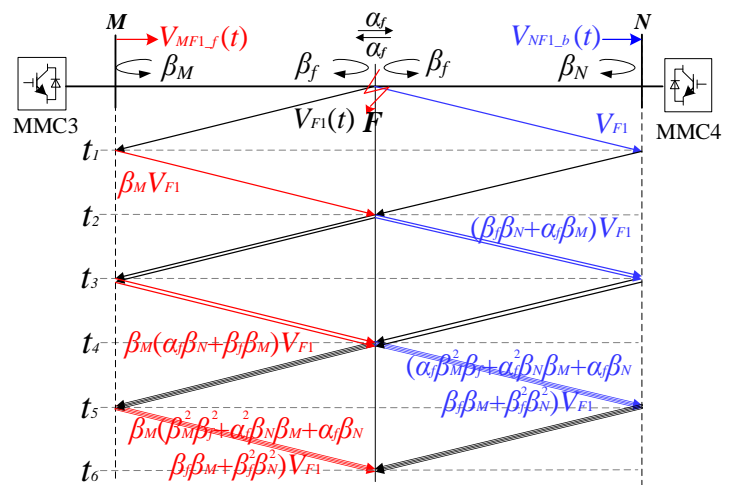

Fig. 3 Traveling wave analysis under internal DC fault.

Table 1 Traveling waves of $V_{M F 1_{-} f}$ and $V_{N F 1_{-} b}$

\begin{tabular}{c|c|c}
\hline \hline $\boldsymbol{t}$ & $\boldsymbol{V}_{\boldsymbol{M F 1} \boldsymbol{f}}$ & $\boldsymbol{V}_{N F 1 \_b}$ \\
\hline \hline $0 \leq t<t_{1}$ & 0 & 0 \\
\hline$t_{1} \leq t<t_{2}$ & $\beta_{M} V_{F 1}$ & $V_{F 1}$ \\
\hline$t_{2} \leq t<t_{3}$ & $\beta_{M} V_{F 1}$ & $V_{F 1}$ \\
\hline$t_{3} \leq t<t_{4}$ & $\beta_{M}\left(1+\alpha_{f} \beta_{N}+\beta_{f} \beta_{M}\right) V_{F 1}$ & $\left(1+\beta_{f} \beta_{N}+\alpha_{f} \beta_{M}\right) V_{F 1}$ \\
\hline$t_{4} \leq t<t_{5}$ & $\beta_{M}\left(1+\alpha_{f} \beta_{N}+\beta_{f} \beta_{M}\right) V_{F 1}$ & $\left(1+\beta_{f} \beta_{N}+\alpha_{f} \beta_{M}\right) V_{F 1}$ \\
\hline \multirow{2}{*}{$t_{5} \leq t<t_{6}$} & $\beta_{M}\left(1+\alpha_{f} \beta_{N}+\beta_{f} \beta_{M}+\beta_{f}^{2} \beta_{M}^{2}+\right.$ & $\left(1+\beta_{f} \beta_{N}+\alpha_{f} \beta_{M}+\alpha_{f} \beta_{M}^{2} \beta_{f}+\right.$ \\
& $\left.\alpha_{f}^{2} \beta_{N} \beta_{M}+\alpha_{f} \beta_{N} \beta_{f} \beta_{M}+\beta_{f}^{2} \beta_{M}^{2}\right) V_{F 1}$ & $\left.\alpha_{f}^{2} \beta_{M} \beta_{N}+\alpha_{f} \beta_{N} \beta_{f} \beta_{M}+\beta_{f}^{2} \beta_{N}^{2}\right) V_{F 1}$ \\
\hline \hline
\end{tabular}

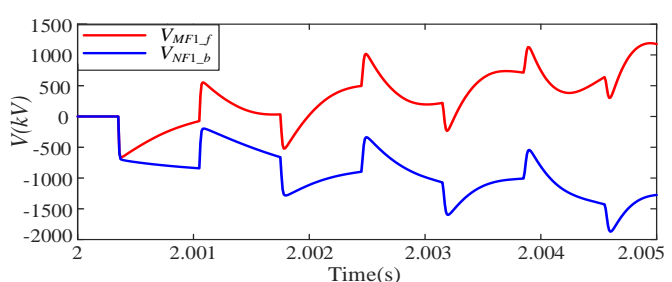

Fig. 4 Illustration of $V_{M F 1_{-} f}$ and $V_{N F 1_{-} b}$ under an internal DC line fault.

\section{B. External DC Line Faults}

When a pole-to-pole DC bus fault occurs, the fault voltage traveling wave will propagate along OHL 34 from the fault point, as shown in Fig. 5.

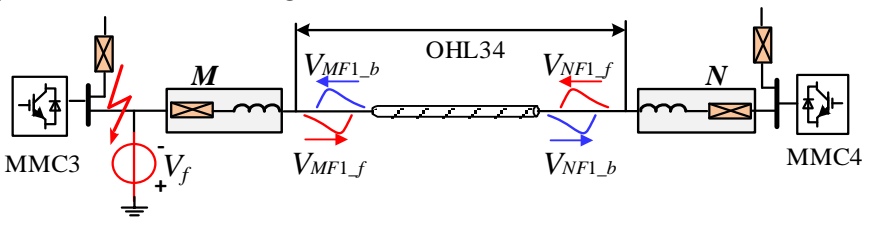

Fig. 5 Equivalent circuit under DC bus fault.

Since there is no fault on OHL 34, the wave impedance does not change. According to the traveling wave transmission theory, the forward line-mode voltage traveling wave $V_{M F 1_{-} f}$ and the backward line-mode voltage traveling wave $V_{N F 1 \_b}$ in $s$ domain satisfies [19],

$$
V_{N F 1_{-} b}(s)=V_{M F 1_{-} f}(s) e^{-\gamma(s) l}
$$

where $\gamma(s)=\sqrt{Z(s) Y(s)}$, representing the propagation constant of the fault traveling wave. $Z(s)$ and $Y(s)$ are the series impedance and parallel admittance of a unit length transmission line, respectively. $l$ is the total length of OHL 34. $e^{-\gamma(s) l}$ represents the propagation function considering attenuation and delay [20]. Supposing a step change of $-500 \mathrm{kV}$ in $V_{M F 1 \_}$, the measured traveling wave $V_{N F 1 \_ \text {b }}$ is depicted in Fig. 6 .

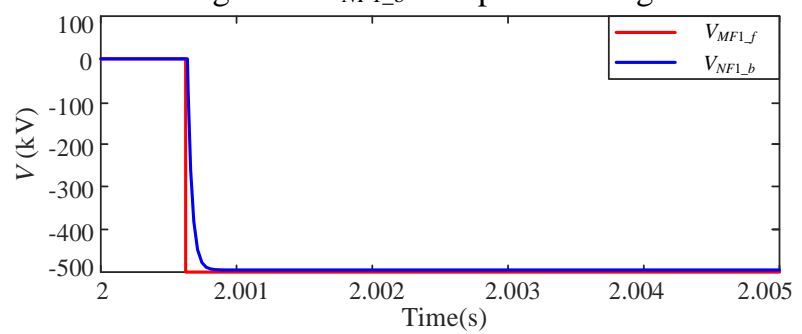

Fig. 6 Illustration of $V_{M F 1 \_f}$ and $V_{N F 1 \_b}$ under an external DC fault.

Fig. 6 shows that the transfer function $e^{-\gamma(s) l}$ only has an attenuation effect, which remains the similarity of the original waveform. Supposing the transmission line is lossless (the distributed resistance and the distributed conductance are both $0)$, equation (4) can be solved as

$$
V_{N F 1_{-} b}(t)=V_{M F 1_{-} f}(t-l / v)
$$

where $v$ is the wave propagation speed. It can be seen from equation (5) that $V_{N F 1_{-} b}$ and $V_{M F 1_{\_} f}$ are identical at different times.

From the above analysis, it can be concluded that the similarity of traveling waves $V_{M F 1 \_f}$ and $V_{N F 1 \_b}$ under external faults is significantly different from that under internal faults. The similarity degree between $V_{N F 1_{-} b}$ and $V_{M F 1_{-} f}$ in the case of an external fault is higher than that under an internal fault. Similarly, $V_{M F 1_{\_} b}$ and $V_{N F 1_{\_} f}$ also have a high degree of 
This paper is a post-print of a paper submitted to and accepted for publication in IEEE Journal of Emerging and Selected Topics in Power Electronics and is subject to Institution of Electrical and Electronic Engineering Copyright. The copy of record is available at IEEE Xplore Digital Library

similarity.

\section{Pole-to-Ground DC Line Fault}

For bipolar DC transmission systems, there is a coupling between the positive and negative poles. When a pole-to-ground (PTG) fault occurs, according to the parallel multi-conductor coupling theory, the fault voltage traveling wave $V_{F_{-} p}$ on the positive pole has the same polarity and similar waveforms compared to the coupling voltage traveling wave $V_{F_{-} n}$ on the negative pole. And they satisfy,

$$
V_{F_{-} n}=\left(Z_{12} / Z_{11}\right) V_{F_{-} p}=k_{12} V_{F_{-} p}
$$

where $Z_{11}$ is the self-impedance of the transmission line. $Z_{12}$ is the mutual impedance between the polar lines. And $k_{12}$ is the coupling coefficient between the lines.

Since $Z_{12}<Z_{11}$ [21], $k_{12}<0.5$. According to equation (6), the voltage drop on the faulty pole line is larger than that of the healthy pole during the pole-to-ground fault.

\section{Design of DifFEREntial Pilot Protection Scheme}

Based on the aforementioned analysis, to reduce the communication burden, only the line-mode voltage is transmitted to each station to design the differential pilot protection.

\section{A. Design of Start-up Element}

As mentioned in Section II, the synchronization error is the timing error amid the protection devices at both ends of the line, which will cause the sampling waveform data to shift in time. The essential reason is that the time-stamp of the sampling data depends on the clock in converter stations. Thus, to avoid the impact of the synchronization error problem on protection, the schemes that the data time-stamp for fault identification does not depend on the station clock are optional, such as the traveling wave startup scheme.

Fig. 7 (a) figures out the waveforms of $V_{M F 1 \_}$and $V_{N F 1 \_b}$ for example. $t_{\text {setup_ } M}$ and $t_{\text {setup_ } N}$ are the arrival time of the fault traveling waves at the $M$ and $N$ sides respectively. By determining the fault traveling wave arrival time $t_{\text {setup_ } M}$ and $t_{\text {setup_ } N}$ and using this time as the zero time of data time-stamp for fault identification on each side, which will be independent of the clock in each station. Subsequently, the data with $t_{w}$ time window is sampled on each side for communication between two stations. The waveforms of the transmitted data are shown in Fig. 7 (b). It can be seen that the time-stamp of the traveling waves data is independent of the clock in stations, avoiding the problem of communication synchronization errors. Even if there is a synchronization error, the proposed scheme is not affected. Thus, there is no need to use the complex schemes e.g. the method proposed in [16], to deal with asymmetrical channel delay or synchronization error.

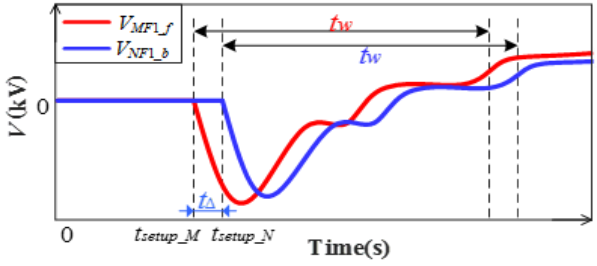

(a) Illustration of fault traveling waves

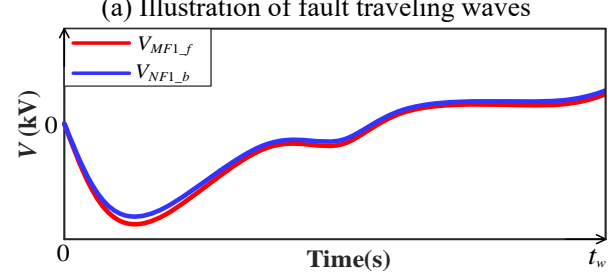

(b) Sampled data for communication

Fig. 7 Protection start-up by determining the arrival time of TWs.

The multi-resolution morphological $(M M)$ algorithm is concerned with the shape and the structure of a signal waveform in time-domain. Since the random communication errors have little effect on the overall waveform structure, $M M$ algorithm has a good performance on waveform information extraction under communication problems. Hence, to detect the arrival time of fault TW quickly and accurately, this paper uses the multi-resolution morphological gradient $(M M G)$ [22][23].

The $M M G$ uses two structural elements $(S E) g^{+}$and $g^{-}$with different origin positions to extract the upper and lower edges of a signal $f$, it can be calculated as:

$$
\operatorname{MMG}(n)=\rho^{-}(n)-\rho^{+}(n)
$$

where $\rho^{+}(n)=\left(f \oplus g^{+}\right)(n)+\left(f \Theta g^{+}\right)(n), \rho^{-}(n)=\left(f \Theta g^{-}\right)(n)+(f$ $\left.\oplus g^{-}\right)(n), n$ is the length of the data window . And " $\oplus$ " and " $\Theta$ " represent the dilation and erosion operators respectively, which are defined as [22]:

$$
\left\{\begin{array}{l}
(f \oplus g)(x)=\max _{y}\{f(x-y)+g(y)\} \\
(f \Theta g)(x)=\min _{y}\{f(x+y)-g(y)\}
\end{array}\right.
$$

where $y \in D_{g}$ (the domain of $g$ ), $x \in D_{f}$ (the domain of $f$ ).

The value of $|\mathrm{MMG}|$ can characterize the geometric abrupt change degree of the waveforms, which can be used to detect the change of waveforms caused by faults [24]. The larger change of the waveform, the larger $|\mathrm{MMG}|$ of this waveform. Besides, the polarity of MMG can reflect the polarity of the change of waveforms. When no fault occurs on the DC line, the traveling wave induced by the fault is always 0 and the calculated $M M G$ is 0 . When faults occur, the protection device will detect a sudden change upon the arrival of fault TW, leading to the change of MMG. Since the fault TW polarity is negative, $M M G<0$. Therefore, the threshold $K_{\text {set }}$ can be set to determine the arrival time of fault TW:

$$
M M G<K_{\text {set }}
$$

To determine $K_{\text {set }}$ appropriately, $\left|K_{\text {set }}\right|$ should be smaller than the $|\mathrm{MMG}|$ in the extreme case that an internal P-PTG fault at one terminal of OHL 34 occurs with high fault resistance. Thus, the calculation of threshold $K_{\text {set }}$ can be expressed as:

$$
K_{\text {set }}=D \times M M G_{\text {Extreme }}
$$

In equation (10), $D$ is the reliability coefficient. It is selected as 0.5 in this paper. $M M G_{\text {Extreme }}$ is the MMG of traveling wave under the extreme case.

B. Fault Identification Based on TW Correlation and Morphological Filtering

Referring to Section III, the correlation between the $M$-side forward line-mode fault voltage TW $V_{M F 1_{-} f}$ and the $N$-side 
This paper is a post-print of a paper submitted to and accepted for publication in IEEE Journal of Emerging and Selected Topics in Power Electronics and is subject to Institution of Electrical and Electronic Engineering Copyright. The copy of record is available at IEEE Xplore Digital Library

backward line-mode fault voltage TW $V_{N F 1 \_b}$ can be used as a criterion for fault identification. Once the protection algorithm is triggered, the line-mode fault voltage TW data is transmitted to the other terminal. However, data errors and data loss can be seen as the random pulse noise, which will distort the waveform. Since the linear filters will destroy the edge and cannot effectively filter out the impulse noise, this paper adopts the nonlinear filter. Among the nonlinear filters, the mathematical morphological filtering $(M F)$ algorithm performs the image process from the perspective of image geometry. The $M F$ algorithm can keep the original image structure from being passivated while filtering. And it has a good suppression effect on the pulse signal. For the waveform $V_{0}$ with impulsive noise, the waveform before and after being processed by the $M F$ algorithm is shown in Fig. 8. Therefore, the protection of each station can use the morphological filtering algorithm to perform data preprocessing on the received waveform data to reduce the impact of abnormal pulses caused by random data error and data loss on the original waveform data during communication.

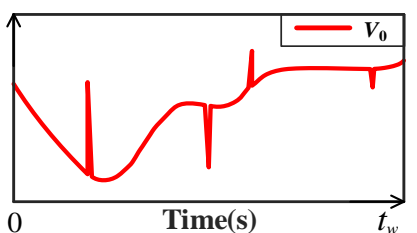

(a) Before $M F$

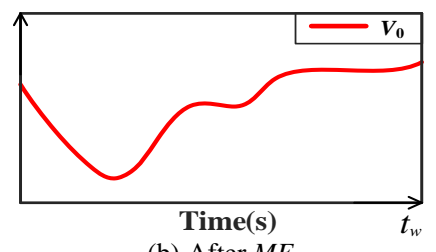

(b) After $M F$
Fig. 8 Suppression of impulsive noise by morphological filtering.

Considering the filtering effect, this paper adopts the Alternate Hybrid Filter $(A H F)$.

$$
\begin{gathered}
A H F=\frac{1}{2} \cdot\left[F_{O C}(f(x))+F_{C O}(f(x))\right] \\
\left\{\begin{array}{l}
F_{O C}(f(x))=((f \circ g) \bullet g)(x) \\
F_{C O}(f(x))=((f \bullet g) \circ g)(x)
\end{array}\right.
\end{gathered}
$$

In equation (12), “॰” and " $\circ$ " represent the opening and closing operators respectively [25]. The opening and closing operations of the structural element $g$ on the original signal $f$ are defined as:

$$
\begin{aligned}
& F_{O}=(f \circ g)(x)=(f \Theta g \oplus g)(x) \\
& F_{C}=(f \bullet g)(x)=(f \oplus g \Theta g)(x)
\end{aligned}
$$

Denote the preprocessed data $\left(V_{M F 1_{-} f}\right.$ and $\left.V_{N F 1_{-} b}\right)$ as two space vectors $x(n)$ and $y(n)$, where $n$ is the length of the data window. The angle cosine correlation coefficient is used to measure the similarity between the two vectors, which is defined as:

$$
r=\frac{\sum_{k=1}^{n}[\boldsymbol{x}(k) \times \boldsymbol{y}(k)]}{\sqrt{\sum_{k=1}^{n}(\boldsymbol{x}(k))^{2} \times \sum_{k=1}^{n}(\boldsymbol{y}(k))^{2}}}
$$

The correlation coefficient $r$ indicates a positive correlation when it is close to 1 , while a negative correlation when it is close to -1 , and no correlation when it is 0 .

From the previous analysis, it can be known that the correlation coefficient between $V_{M F 1_{-} f}$ and $V_{N F 1_{-} b}$ during an external fault is larger than that during an internal fault. Thus, the fault identification criterion is designed as shown in (15), where $r_{\text {set }}$ is the setting value.

$$
\left\{\begin{array}{l}
r<r_{\text {set }}, \text { Internal fault } \\
r>r_{\text {set }}, \text { External fault }
\end{array}\right.
$$

The relationship of correlation coefficient $r$ and the similarity degree is concluded in Table 2. Referring to the analysis in Section III.A and III.B, the similarity of waveforms is strong under an external fault, and weak under an internal fault. Thus, the threshold $r_{\text {set }}$ can be set as 0.5 to leave a certain margin.

Table 2 Relationship of correlation coefficient $r$ and degree of similarity

\begin{tabular}{c||c}
\hline Correlation coefficient $r$ & Degree of similarity \\
\hline \hline $0.8-1.0$ & very strong \\
$0.6-0.8$ & strong \\
$0.4-0.6$ & medium \\
$0.2-0.4$ & weak \\
$-1.0-0.2$ & very weak \\
\hline
\end{tabular}

\section{Faulted Pole Discrimination based on MMG Ratio}

According to Section III.C, the voltage drop of the positive and negative poles can be used to discriminate the faulted poles. The $M M G$ introduced earlier can characterize the degree of the geometric abrupt change of the pole voltage waveforms, which can be used to evaluate the pole voltage drops. After protection starts, the minimum values of the $M M G$ of the positive and negative pole voltages are denoted as $M M G_{P}$ and $M M G_{N}$, respectively. This paper introduces the $M M G$ ratio $d=M M G_{P} / M M G_{N}$ and the faulted pole discrimination criterion is designed as shown in (16), where $\lambda$ is the setting value.

$$
\begin{cases}d>\lambda & , \text { Positive pole to ground }(P-P T G) \\ 1 / \lambda \leq d \leq \lambda, & \text { Pole to pole }(P T P) \\ d<1 / \lambda, & \text { Negative pole to ground }(N-P T G)\end{cases}
$$

According to equation (6), $k_{12}<0.5$, which means that the fault voltage traveling wave $V_{F \_}$is larger than the coupling voltage traveling wave $V_{F_{n} n}$. Assuming that $\mathrm{MMG}$ is approximately proportional to the amplitude change and considering $k_{12}<0.5, d$ will be larger than 2 under a fault on the positive pole and less than 0.5 under a fault on the negative pole line. Thus, $\lambda$ can be set as 2 to leave a certain margin.

\section{Overall Scheme of the Differential Pilot Protection}

To be concluded, the overall resilient-oriented differential pilot protection scheme is depicted in Fig. 9.

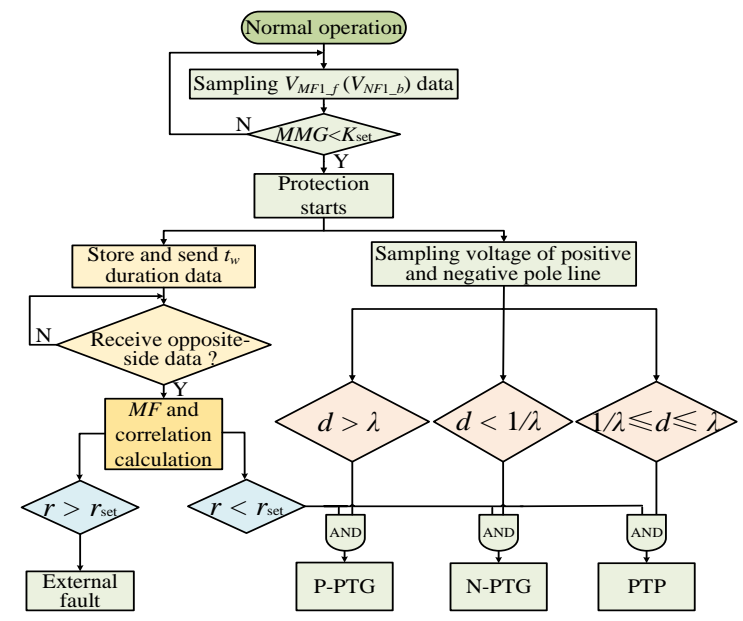

Fig. 9 Flowchart of the proposed differential pilot protection. 
This paper is a post-print of a paper submitted to and accepted for publication in IEEE Journal of Emerging and Selected Topics in Power Electronics and is subject to Institution of Electrical and Electronic Engineering Copyright. The copy of record is available at IEEE Xplore Digital Library

In Fig. 9, the morphological gradient algorithm is used to determine the arrival time $t_{\text {setup_M }}, t_{\text {setup_ } N}$ of the fault traveling waves on each terminal. The $M$-side $(N$-side) protection calculates $M M G$ of the forward (backward) line-mode traveling wave of the fault voltage during the sliding time window. If the calculated $M M G$ is less than $K_{\text {set }}$, the fault traveling wave arrives and the protection algorithms trigger. At the same time, the data within $t_{w}$ time window after protection starts is stored and sent to the opposite side. After receiving the data from the opposite side, the protection uses the morphological filtering algorithm to preprocess the waveform data affected by the communication problems. Subsequently, the waveform correlation coefficient is calculated. If the correlation coefficient is larger than $r_{s e t}$, an external fault is deemed to occur. If the correlation coefficient is less than $r_{\text {set }}$, it is considered to be an internal fault. The faulted poles are identified by using the morphological gradient ratio $d$. Once an internal DC line fault happens, the DC circuit breakers (DCCB) on the faulted line poles will be tripped to isolate the fault line. If the fault is external, the DCCBs on both sides will not operate.

\section{Simulation VerificAtion}

To test the effectiveness of the proposed differential pilot protection, a $\pm 500 \mathrm{kV}$ four-terminal bipolar MMC based DC grid shown in Fig. 1 is built in PSCAD/EMTDC, where MMC4 controls the DC voltage and MMC1-MMC3 control the transmitted power. The active power transmitted by MMC1MMC3 to the DC grid is 0.95p.u., 0.95p.u. and -0.95p.u., respectively. The transmission overhead line adopts the frequency-dependent model. The other parameters are listed in Table 3.

Since the multiplexed fiber configuration is conventionally adopted for transmission distances larger than 50km [26], the multiplexed optical fiber configuration is considered in this paper. The structure diagram is depicted in Fig. 10. Where the multiplexed optical fiber communication system includes two channels (coaxial cable and optical cable). Since the propagation characteristics of the binary electric pulse signals in different channels are different, each channel must choose the code pattern (electric pulse waveform) suitable for this channel. In practical engineering, the NRZ (Non-Return-to-Zero) code pattern is used for the optical fiber channel. While for the cable channel, the HDB3 (High-Density Bipolar of Order 3) code pattern is used due to that the main lobe of the power spectrum of the HDB3 code pattern is more concentrated [26][27]. In order to focus on the data communication problem and simplify the modeling process, this paper ignored the data errors in the fiber pigtail channel and the cable channel, and only studied the data problems in long-distance fiber communication between stations.

\begin{tabular}{c||cccc} 
Table 3 & Parameters of MMC based DC grid \\
\hline MMC & 1 & 2 & 3 & 4 \\
\hline \hline AC voltage /kV & 220 & 220 & 500 & 500 \\
Rated power /MW & 1500 & 1500 & 3000 & 3000
\end{tabular}

\begin{tabular}{c||cccc} 
Arm inductance $/ \mathrm{mH}$ & 160 & 160 & 80 & 80 \\
Number of SMs per arm $/ \mathrm{N}$ & 122 & 122 & 122 & 122 \\
Sub-module capacitance $/ \mathrm{mF}$ & 10 & 10 & 15 & 15 \\
Rated sub-module voltage $/ \mathrm{kV}$ & 4.098 & 4.098 & 4.098 & 4.098 \\
Current limiting inductor/mH & 150 & 150 & 150 & 150 \\
Inductor on ground line $/ \mathrm{mH}$ & 300 & 300 & 300 & 300 \\
\hline
\end{tabular}

The overall communication model is built in MATLAB/ Simulink as shown in Fig. 11 [27]. Where the pulse code modulation (PCM) adopts the A-law 13 polyline 8-bit encoding to generate digital baseband signals. Thus, the sampling frequency of the signal sampler in MATLAB/Simulink model is $8 \mathrm{kHz}$. Since the communication equipment needs to sample the fault waveforms collected by electrical equipment, the sampling frequency of electrical equipment must be no less than $8 \mathrm{kHz}$ of the communication sampling frequency. Thus, the simulation step in PSCAD is selected as $100 \mu \mathrm{s}(10 \mathrm{kHz})$.

In addition, to consider the data problems in the communication process more realistically, the conversion of code patterns between different channels in optical fiber communication needs to be modeled. After the digital signal is pattern-converted at both ends of the coaxial cable, the binary symmetrical channel (BSC) is used to model the optical fiber communication channel. The communication between the sending and receiving terminals is realized by a digital signal serial communication structure. By controlling the bit error rates, different degrees of fiber data errors can be simulated. Moreover, it should be pointed out that this paper only considered the external characteristics of the effect of the communication model on data, and ignored the communication equipment delay and the specific influencing factors such as the characteristics of the dielectric material in optical fiber.

The structural elements ( $S E$ ) are needed in the MMG and $M F$ algorithms [28][29]. For the MMG, $S E$ is used to extract the ascending and descending edges of the transient signal. While for $M F, S E$ is used to filter out the impulse noise of waveforms without changing the original structure of the waveforms. The $S E$ can be expressed as $g=g\left\{g_{1}, g_{2}, g_{3}, \ldots, g_{l-l}, g_{l}\right\}$, where $l$ is the width of $S E$. The $S E s$ in power system applications are often selected as flat structures, thus, $g_{l}=g_{2}=\ldots=g_{l}$. The selection of $S E$ width needs to consider the sampling frequency of the model and the number of sampled data. The sampling frequency in the simulation model in this paper is $10 \mathrm{kHz}$. Considering the reliability of the proposed protection scheme with $10 \mathrm{kHz}$ sampling frequency, the $3 \mathrm{~ms}$ data window is selected. Thus, there are 31 samples within the time window. For MMG, the shorter the width of the flat structure $S E$, the stronger ability to detect disturbances MMG has. Thus, the width of the flat structure $S E$ adopted to calculate MMG is 2 . And $g=\{0,0\}$ can be selected. Considering the influence of communication, the duration of data error and data loss are both 2 sampling periods. For $M F$, due to the width of flat structure, $S E$ must be larger than the width of pulse noise. Thus, the width of $S E$ of the $M F$ algorithm is selected as $l=3$, i.e., $g(x)=\{0,0,0\}$. 
This paper is a post-print of a paper submitted to and accepted for publication in IEEE Journal of Emerging and Selected Topics in Power Electronics and is subject to Institution of Electrical and Electronic Engineering Copyright. The copy of record is available at IEEE Xplore Digital Library

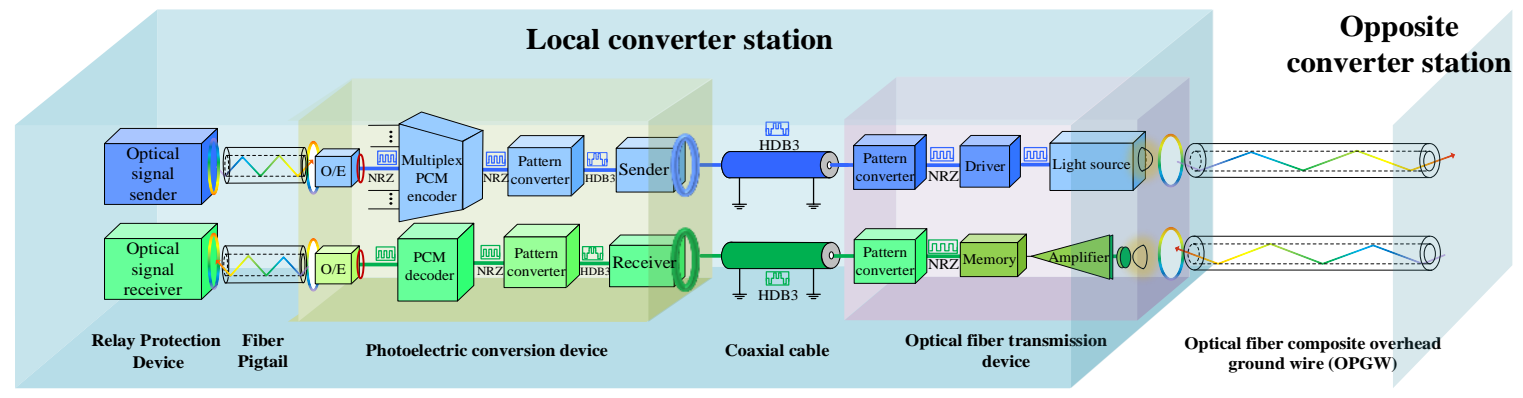

Fig. 10 The structure diagram of multiplexed optical fiber communication system.

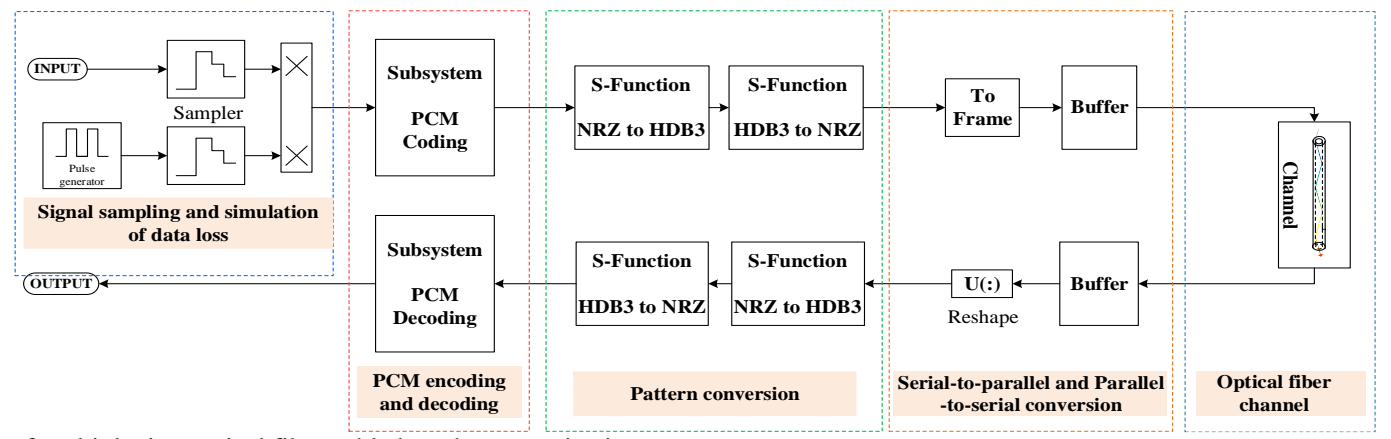

Fig. 11 Modeling of multiplexing optical fiber cable based communication system.

\section{A. Threshold Setting of Start-up Element}

Denoting the DC line faults happened at $0 \mathrm{~km}, 105 \mathrm{~km}, 210$ $\mathrm{km}$ of OHL 34 are $F_{1}, F_{2}$ and $F_{3}$. The faults $F_{4}$ and $F_{5}$ respectively represent the AC fault at MMC3 AC terminal and the DC bus fault at MMC4. To determine $K_{\text {set }}$ appropriately, the extreme situation that an internal P-PTG fault with $1000 \Omega$ resistance occurs at one terminal of OHL 34 is considered and simulated. According to the simulation results, the MMG under the extreme case is $M M G_{\text {Extreme }}=-9.73$. Thus, from equation (10), $K_{\text {set }}$ can be obtained as: $K_{\text {set }}=D \times M M G_{\text {Extreme }}=0.5 \times(-9.73)$ $\approx-5$.

To verify the threshold of the start-up element, the positive PTG and PTP faults with different fault resistances are applied at these fault locations. Taking the protection at MMC3 as an example, Fig. 12 shows the absolute values of the first non-zero point of the calculated $M M G$ waveform of $V_{M F 1_{-} f}$. It can be seen that the absolute value of $M M G$ decreases with the increase of fault resistance and fault distance. As shown in Fig. 12, the $|\mathrm{MMG}|$ under internal faults are all larger than $\left|K_{\text {set }}\right|$. Thus, $K_{\text {set }}=-$ 5 is appropriate, and the protection can start up with sufficient sensitivity.

As can be seen in Fig. 12, under some external faults, the calculated $|\mathrm{MMG}|$ is also large than $\left|K_{\text {set }}\right|$. Thus, the start-up element cannot guarantee fault identification between internal and external faults. Further identification algorithms need to be developed.

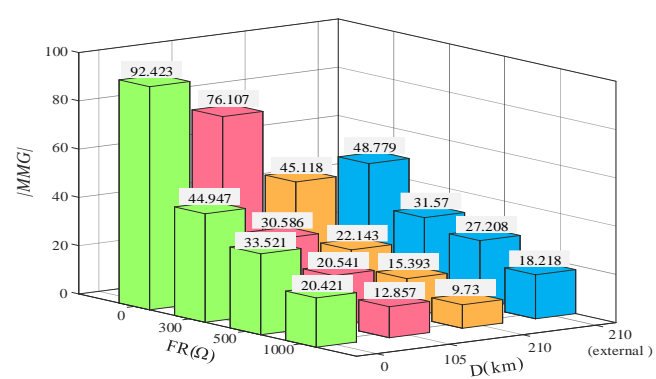

(a) Calculated $|M M G|$ under P-PTG faults.

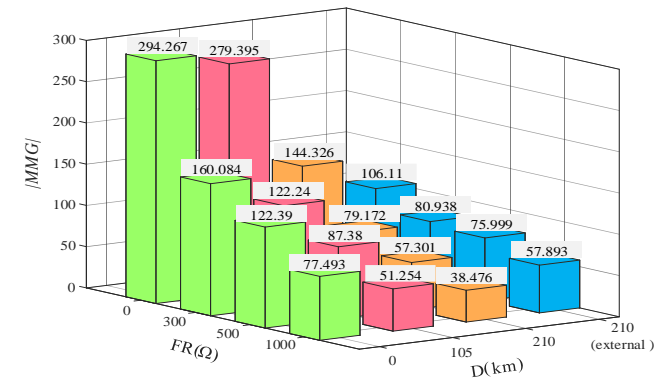

(b) Calculated $|\mathrm{MMG}|$ under PTP faults.

Fig. 12 Calculated |MMG| under different fault resistances and fault distances.

\section{B. Threshold Verification of Fault Identification Criterion}

As mentioned in Section IV, the threshold $r_{\text {set }}$ is set as 0.5, Thus, the fault identification criterion can be expressed as:

$$
\left\{\begin{array}{l}
r>0.5, \text { Internal fault } \\
r<0.5, \text { External fault }
\end{array}\right.
$$

To verify the threshold $r_{\text {set }}$, the positive pole-to-ground faults (P-PTG) and pole-to-pole faults at $F_{2}, F_{3}$, and $F_{5}$ are scanned. And the correlation coefficients of line-mode fault voltage traveling waves $V_{M F 1 \_f}$ and $V_{N F 1 \_b}$ are calculated. Setting the correlation coefficient $r$ as the radius, Fig. 13 shows the calculation results of the correlation coefficients against faults with different fault resistances. It can be seen that the correlation coefficients under internal faults (at $F_{2}$ and $F_{3}$ ) are all within [- 
This paper is a post-print of a paper submitted to and accepted for publication in IEEE Journal of Emerging and Selected Topics in Power Electronics and is subject to Institution of Electrical and Electronic Engineering Copyright. The copy of record is available at IEEE Xplore Digital Library

1, 0.5]. While under external fault (at $F_{5}$ ), the correlation coefficients of the two waveforms are close to 1 , as shown in the blue line in Fig. 13. Hence, $r_{\text {set }}=0.5$ is appropriate.

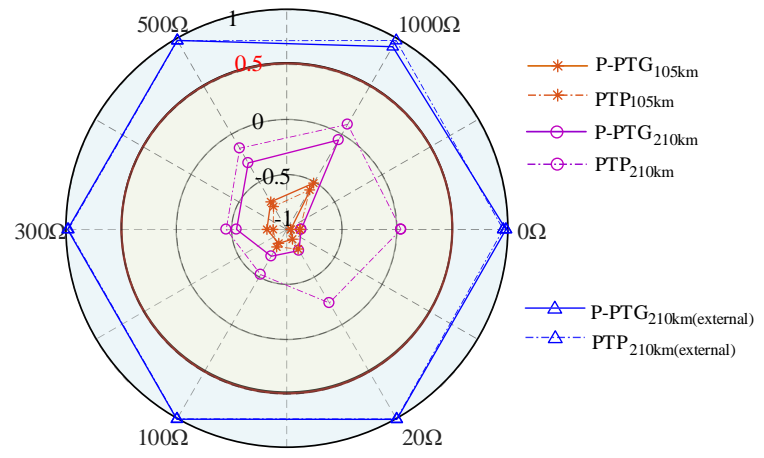

Fig. 13 Calculation results of correlation coefficients.

C. Threshold Verification of Faulted Pole Discrimination Criterion

According to the analysis in Section III.C, the faulted pole can be determined by comparing the magnitude of $M M G$ of the voltage on the positive and negative poles. Fig. 14 shows the ratio of $M M G$ of the positive and negative voltages under different faults at $F_{2}$. It can be seen that the ratio of $M M G$ under different fault types has obvious differences, and it is hardly affected by the fault resistances. Referring to Fig. 14, when $\lambda$ is set to 2 , the protection can effectively identify different fault types.

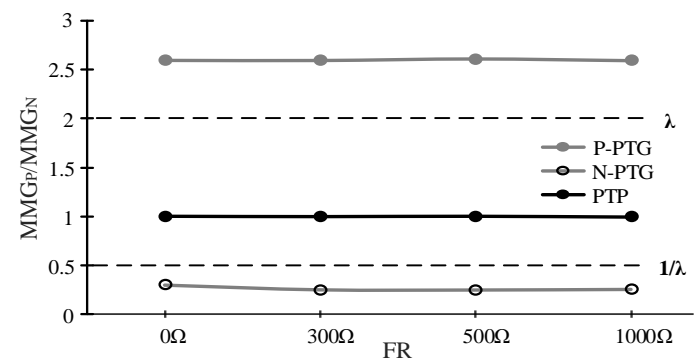

Fig. 14 The ratio of $M M G$ under different fault types and fault resistances.

\section{Performance of Fault Traveling Wave Location}

To test the performance of the effectiveness of the start-up element and the location of fault traveling wave arrival time, a positive PTG fault with $300 \Omega$ resistance is applied at $180 \mathrm{~km}$ away from MMC3 at $2.0 \mathrm{~s}$. Fig. 15 shows that the arrival time of the line-mode fault traveling waves $V_{M F 1_{-} f}$ and $V_{N F 1_{-} b}$ is located by detecting the $M M G<-5$ within the data window.

The simulation results in Fig. 15 show that the arrival time (blue and red dotted lines) of the line-mode fault voltage TWs of converter stations on both sides of OHL34 are $t_{M M C 4}=2.0001 \mathrm{~s}$ and $t_{M M C 3}=2.0006 \mathrm{~s}$, respectively. The arrival time (at the blue and red rectangular labels) detected by $M M G$ are $t_{\text {setup_ } N}=2.0001 \mathrm{~s}$ and $t_{\text {setup_ } M}=2.0006 \mathrm{~s}$ respectively. And the corresponding values of $M M G$ are -60.51 and -71.46 . Simulation results show that $M M G$ can be used to accurately locate the fault traveling waves at both sides of the DC line.

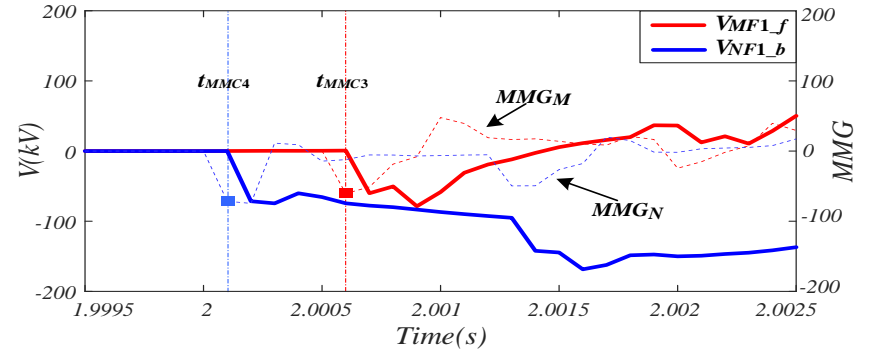

Fig. 15 Simulation results of fault traveling wave location.

E. Performance of Fault Identification in the DC grid

The topology of the four-terminal meshed DC grid is simplified as shown in Fig. 16. $F_{6}$ is the midpoint of OHL13, where a PTP fault occurs at $2 \mathrm{~s} . P_{i j}$ is the protection device on the $\mathrm{MMC}_{i}$ side of $\mathrm{OHL}_{i j}$. The correlation coefficients between the forward traveling wave $V_{i j f}$ and the backward traveling wave $V_{j i-b}$ are used to be the criterion of the protection on $\mathrm{OHL}_{i j}$.

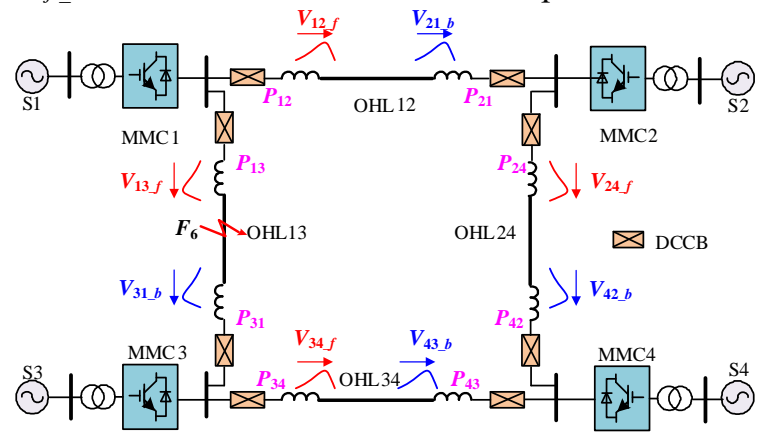

Fig. 16 Topology of the four-terminal meshed DC grid (simplified view).

The waveforms of the traveling waves used to calculate correlation coefficients are shown in Fig. 17. It can be seen that the two traveling waves on the healthy OHLs (OHL12, OHL24, OHL34) are much similar than those on the faulty OHL (OHL13).
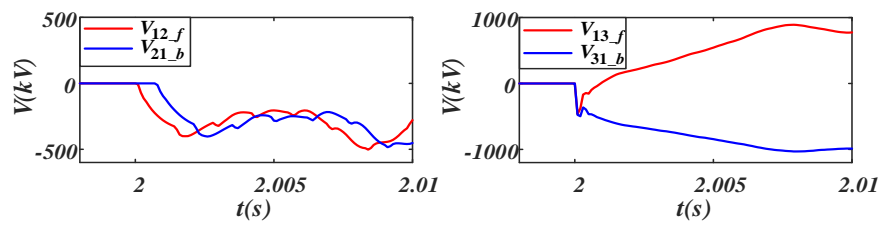

(a) OHL 12
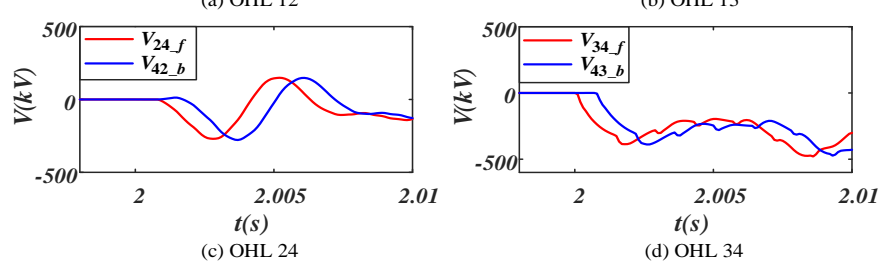

Fig. 17 The waveforms of the traveling waves on different OHLs.

By calculating the correlation coefficient $r$, the protection devices can identify whether there is an internal fault or not. The identification results of protection devices on different OHLs are shown in Table 4.

Table 4 The identification result of the protection devices on different OHLs.

\begin{tabular}{c||c|c|c}
\hline $\begin{array}{c}\text { Different } \\
\text { lines }\end{array}$ & $\begin{array}{c}\text { Correlation } \\
\text { coefficient } r\end{array}$ & $\begin{array}{c}\text { Threshold } \\
r_{\text {set }}\end{array}$ & $\begin{array}{c}\text { Identification } \\
\text { result }\end{array}$ \\
\hline \hline OHL 12 & 0.976 & 0.5 & Healthy \\
\hline OHL 13 & -0.692 & 0.5 & Faulty \\
\hline OHL 24 & 0.987 & 0.5 & Healthy \\
\hline OHL 34 & 0.981 & 0.5 & Healthy \\
\hline
\end{tabular}


This paper is a post-print of a paper submitted to and accepted for publication in IEEE Journal of Emerging and Selected Topics in Power Electronics and is subject to Institution of Electrical and Electronic Engineering Copyright. The copy of record is available at IEEE Xplore Digital Library

From Table 4, it can be found that the correlation coefficients of healthy OHLs are much larger than the threshold. While the correlation coefficient of the faulty OHL is much less than the threshold. Thus, with the proposed protection scheme, the protection devices on each transmission line of the DC grid can correctly identify internal or external faults.

\section{RoBUSTNESS ANALYSIS}

\section{A. Robustness to Data Errors and Data Loss}

The requirements of the BER index in optical fiber communication for power line protection are proposed in the standard [30]. Considering that the communication time of the scheme proposed in this paper is less than $1 \mathrm{~s}$, the $1 \%$ BER is used as the threshold for severe data errors. At present, there is no standard for the degree of data loss in optical fiber communication. Thus, this paper tests the performance of the proposed scheme to tolerate communication failure in the case of $5 \%$ data loss.

To test the performance of morphological filtering $(M F)$ preprocessing on communication failures, a positive PTG fault with $300 \Omega$ fault resistance is applied at $F_{2}$ (internal fault). The communication model in MATLAB/Simulink is used to simulate the $V_{N F 1 \_b}$ data sent from MMC4 to MMC3 under normal communication, $1 \%$ bit error rate (BER), $1 \%$ bit error rate, and $2(5 \%)$ data loss respectively. Fig. 18 shows the processing performance of $M F$ under different communication failures.

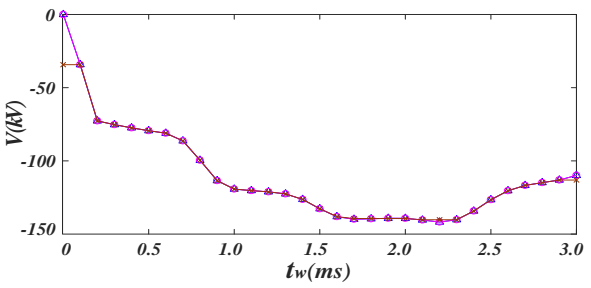

(a) Normal communication

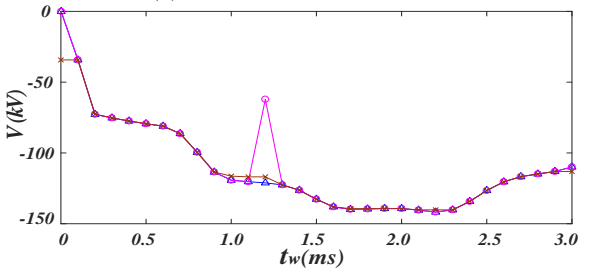

(b) $1 \%$ bit error rate (BER)

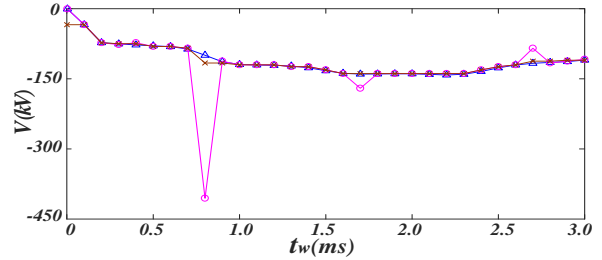

(c) $1 \%$ bit error rate

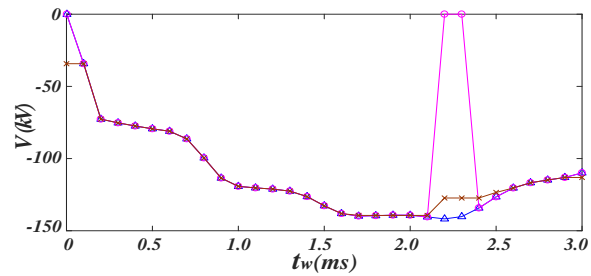

(d) $2(5 \%)$ data loss $\triangle$
Fig. 18 Morphological filtering effect of $V_{N F I_{-} b}$ data under different communication failures.

In Fig. 18, $V_{N F I_{-} b}$ is the sampled line-mode fault TW at MMC 4. $V_{N \_ \text {сотти }}$ is the waveform received at MMC3 through the optical fiber communication. $V_{N \_M F}$ is the pre-processed waveform using the morphological filter at MMC3. As can be seen from Fig. 18 (b)-(d), the impact of data error and data loss on the original waveform can be regarded as impulsive noise. But the morphological filtering can effectively filter out these impulsive noises. Even when the bit error rate is as high as $1 \%$ and two data are continuously lost, the protection can still be guaranteed to operate reliably.

Fig. 19 shows the calculation results of the correlation coefficients before and after morphological filtering under different internal fault resistances and communication failures. It can be seen that even without morphological filtering, the correlation coefficients under different communication problems can still be kept below the threshold value of 0.5 . This is because that the $V_{N F 1 \_b}$ waveform becomes more cluttered under data errors and data loss, which makes the similarity between the $V_{N F 1 \_b}$ and $V_{M F 1 \_f}$ waveforms weaker. From the definition of the correlation coefficient, it can be seen that the correlation coefficient under $1 \%$ BER will be around 0 , which is still less than 0.5 , and will not cause protection to refuse to start. Fig. 19 also shows that after morphological filtering, the distribution of correlation coefficients is closer to that under normal communication. This is because the original correlation features are 'restored' after the morphological filtering process.

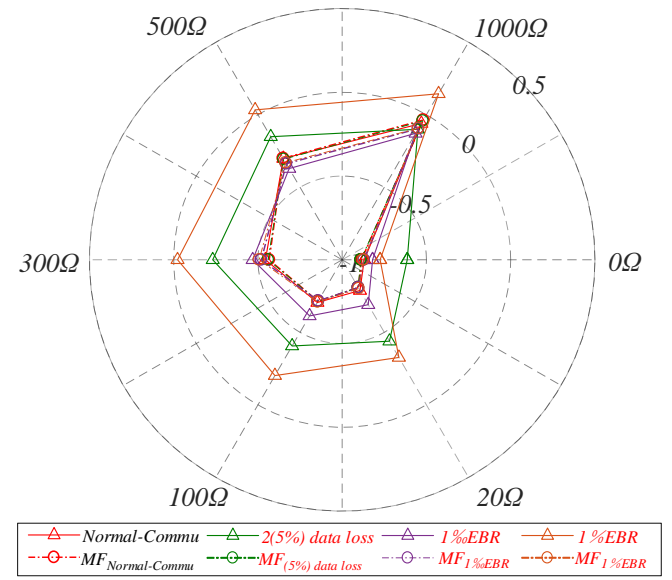

Fig. 19 Correlation coefficients before and after filtering under different communication problems.

As for the external faults, apply positive pole to ground faults at $F_{5}$. Fig. 20 shows the correlation coefficients under different communication problems. It can be seen that both data loss and data error will cause the correlation coefficients to decrease. The more serious the data error is, the larger decrease in the correlation coefficient. In the case of $1 \%$ BER, the correlation coefficient will be reduced to less than 0.5 during a metallic PTG fault, which will cause protection malfunction. Fig. 20 also shows that after the morphological filtering is performed on the communication data $V_{N F 1 \_b}$, the calculation results of the correlation coefficients are close to the correlation coefficients 
This paper is a post-print of a paper submitted to and accepted for publication in IEEE Journal of Emerging and Selected Topics in Power Electronics and is subject to Institution of Electrical and Electronic Engineering Copyright. The copy of record is available at IEEE Xplore Digital Library

during normal communication. After preprocessed by morphological filtering, the waveform data affected by the communication problems are recovered. To conclude, the data errors and data loss of the $V_{N F 1 \_b}$ waveform will reduce the correlation with the $V_{M F 1_{-} f}$ waveform, which may cause maloperation. However, after morphological filtering, the correlation coefficients 'restore' to nearly the normal value.

In summary, it can be seen that whether there is an internal or external fault, the protection scheme proposed in this paper can effectively identify the fault and 'restore' the waveform affected by communication, which makes the correlation coefficients close to those under normal communication. If the measures are adopted, mal-operation of protection schemes may happen under communication failures.

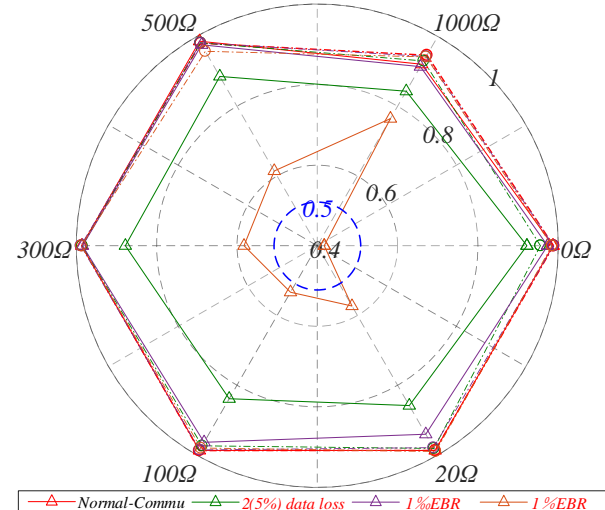

$\triangle$ Normal-Commu $\triangle-2(5 \%)$ data loss $\triangle-T$ \%oEBR $\triangle-1 \%$ EBR

Fig. 20 Correlation coefficients before and after filtering under different communication problems.

\section{B. Robustness to Noise Interference}

To investigate the performance under noise interference, an internal P-PTG fault with $100 \Omega$ resistance and an external PPTG with $300 \Omega$ resistance are applied at $F_{2}$ and $F_{5}$, respectively. The simulation results considering the Gaussian noise of different SNR are shown in Fig. 21. Where, $V_{M F 1_{-} f}$ and $V_{N F 1_{-} b}$ are the forward line-mode fault TW at relay $M$ and the backward line-mode fault TW at relay $N$, respectively. $V_{M \_n o i s e}$ and $V_{N_{-} n o i s e}$ are the measured $V_{M F I_{-} f}$ and $V_{N F I_{-} b}$ affecting by noise interference. $V_{M_{-} M F}$ and $V_{N_{-} M F}$ are the pre-processed voltages of $V_{M \_n o i s e}$ and $V_{N \_n o i s e}$ using $M F$. The correlation coefficients before and after $M F$ under different noise are shown in Fig. 22.
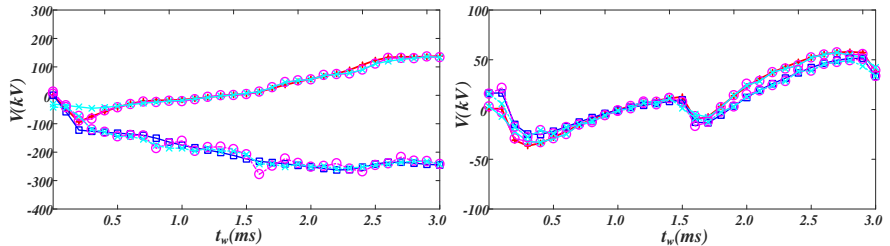

(a) voltages under an internal fault $\mathrm{F}_{2}$ with 100 resistance $(\mathrm{SNR}=20 \mathrm{~dB})$

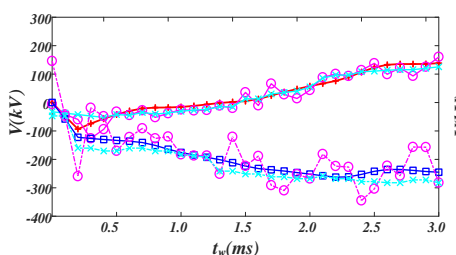

(b) voltages under an internal fault $\mathrm{F}_{5}$ with 300 resistance $(\mathrm{SNR}=20 \mathrm{~dB})$

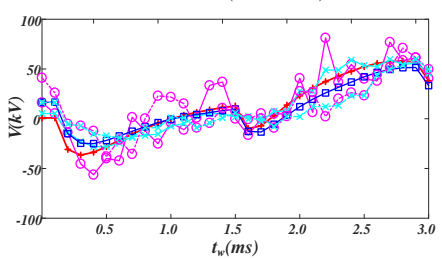

(c) voltages under an internal fault $\mathrm{F}_{2}$ with 100 resistance $(\mathrm{SNR}=10 \mathrm{~dB})$

(d) voltages under an internal fault $\mathrm{F}_{5}$ with 300 resistance $(\mathrm{SNR}=10 \mathrm{~dB})$

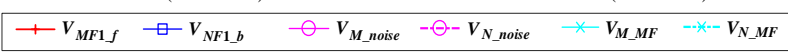

Fig. 21 Simulation result of the scheme in this manuscript under different Gaussian noise.

As shown in Fig. 21 and Fig. 22, although there is a lot of noise in the sampled data, which may deteriorate the performance of protection, the $M F$-preprocessed scheme proposed in this paper can improve the endurance to noise interference. From Fig. 22, it can be found that the protection scheme will not malfunction even under the noise of $10 \mathrm{~dB}$.

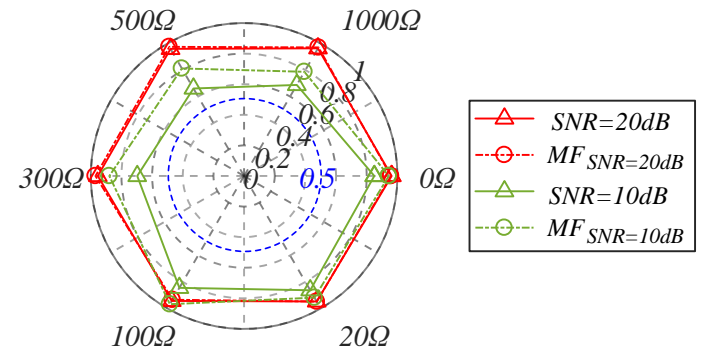

(a) an external fault occurs at $F_{5}$ with different fault resistances

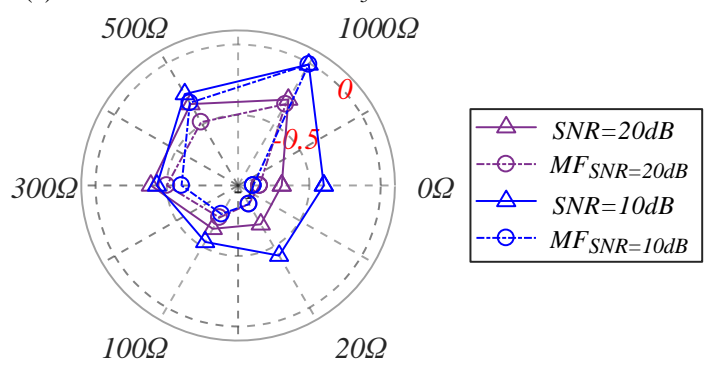

(b) an internal fault occurs at $F_{2}$ with different fault resistances Fig. 22 Correlation coefficients before and after $M F$ under different noise levels.

\section{Performance under Combined Communication Problems}

In this sub-section, the effect of combined communication problems (data errors/loss and noise) on the correlation coefficients is studied. According to the results shown in Fig. 19 and Fig. 22, it can be found that the noise and data errors/loss cannot affect the identification of internal faults. This is due to that the waveform will be more cluttered under data errors/loss and noise, which makes the similarity between the $V_{M F 1 f}$ and $V_{N F 1 \_b}$ waveforms weaker. Thus, the combined effects of noise and data errors/loss under internal faults are not considered here. A P-PTG fault considering the combined problems is applied at $F_{5}$. The simulation waveforms and the calculated 
This paper is a post-print of a paper submitted to and accepted for publication in IEEE Journal of Emerging and Selected Topics in Power Electronics and is subject to Institution of Electrical and Electronic Engineering Copyright. The copy of record is available at IEEE Xplore Digital Library

correlation coefficients before and after $M F$ are shown in Fig. 23 and Fig. 24, respectively.

As can be seen, the impact of combined problems is generally greater than that of single problems. Especially under the problem of " $10 \mathrm{Db}$ noise+2 data loss $+1 \%$ BER", the correlation coefficient will reduce to 0.503 , which is slightly larger than the threshold 0.5 , endangering the reliability of protection. However, it can be found the $M F$ is still efficient to restore the waveforms and beneficial to improve the reliability of protection.

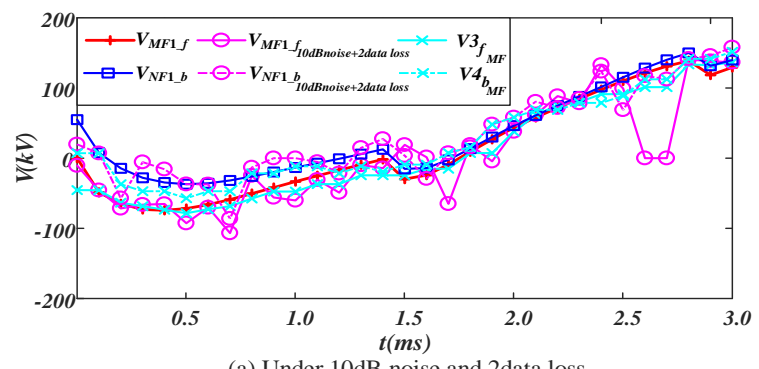

(a) Under $10 \mathrm{~dB}$ noise and 2data loss

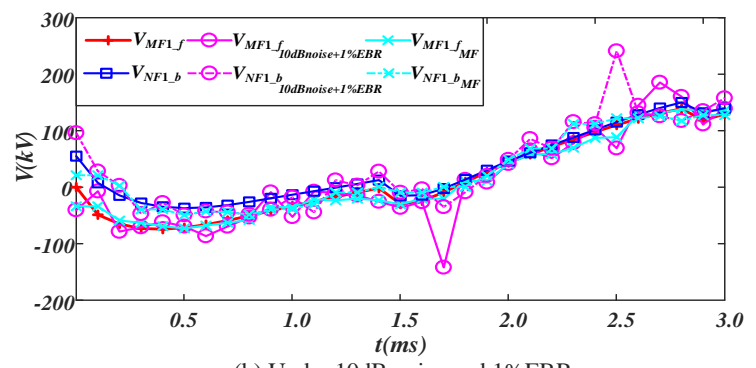

(b) Under $10 \mathrm{~dB}$ noise and $1 \% \mathrm{EBR}$

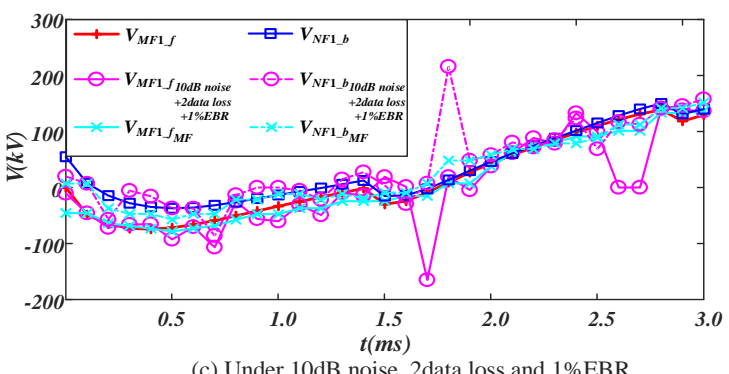

(c) Under $10 \mathrm{~dB}$ noise, 2 data loss and $1 \% \mathrm{EBR}$

Fig. 23 Simulation waveforms under a P-PTG fault considering different combined communication problems.

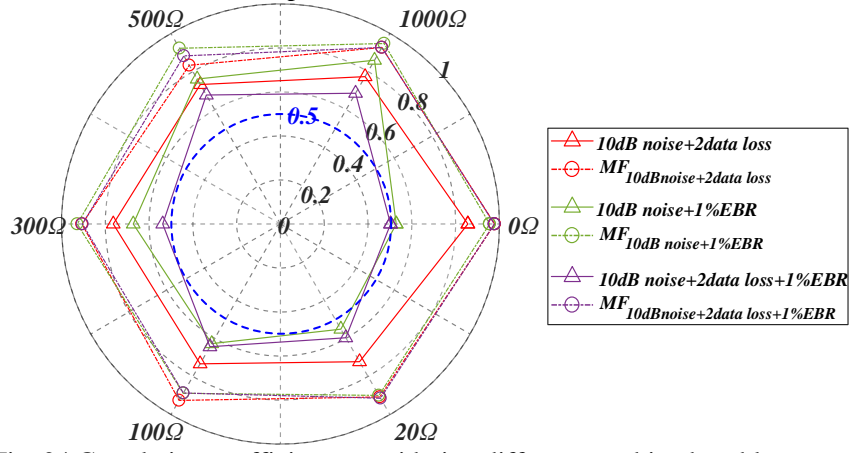

Fig. 24 Correlation coefficients considering different combined problems.

\section{Robustness to Different Sizes of DC Current-Limiting Inductances}

To study the effect of current-limiting inductances (CLIs) on the selection of threshold $r_{\text {set }}$, further simulations considering
CLIs within the range of $50-200 \mathrm{mH}$ have been conducted. The simulation results under PTP faults with $100 \Omega$ resistance occurring at $F_{5}$ and $F_{2}$ are shown in Fig. 25. The corresponding correlation coefficients of these simulation cases are calculated in Table 5.

As shown in Fig. 25 and Table 5, the size of CLI will affect the shape of the waveforms. But the similarity between waveforms $V_{M F I_{f} f}$ and $V_{N F I_{-} b}$ is not affected. Thus, the selection of threshold $r_{\text {set }}$ is robust to different sizes of CLIs.

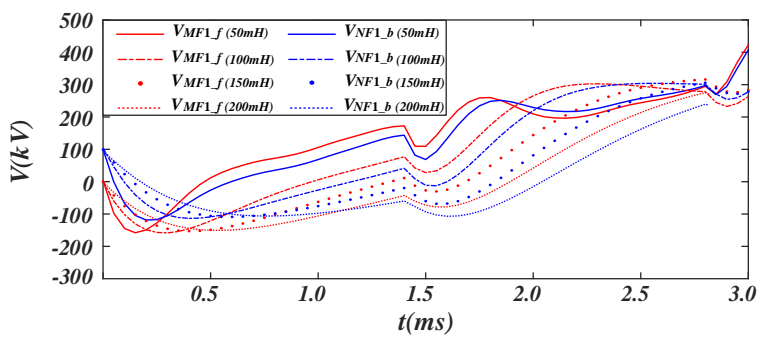

(a) Transient voltages under a PTP fault with $100 \Omega$ resistance at $F_{5}$

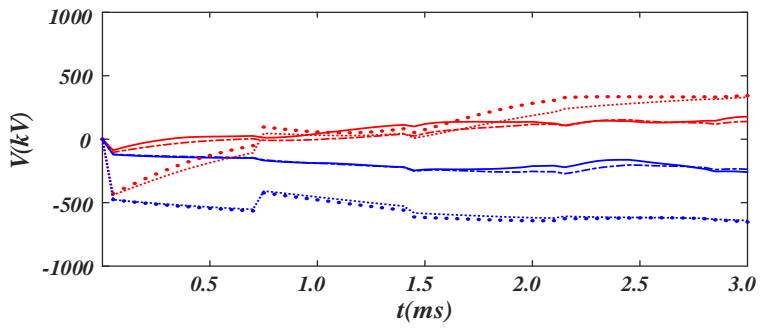

(b) Transient voltages under a PTP fault with $100 \Omega$ resistance at $F_{2}$ Fig. 25 The simulation results considering different CLIs.

Table 5 The correlation coefficients $r$ under different simulation cases.

\begin{tabular}{c||c|c}
\hline \multirow{2}{*}{\multicolumn{1}{c||}{ The size of CLI }} & \multicolumn{2}{c}{ Correlation coefficient $r$} \\
\cline { 2 - 3 } & Internal PTP fault at $F_{2}$ & External PTP fault at $F_{5}$ \\
\hline $50 \mathrm{mH}$ & -0.737 & 0.967 \\
\hline $100 \mathrm{mH}$ & -0.692 & 0.964 \\
\hline $150 \mathrm{mH}$ & -0.473 & 0.961 \\
\hline $200 \mathrm{mH}$ & -0.598 & 0.944 \\
\hline
\end{tabular}

E. Time Delay Evaluation of the Protection Scheme

In this paper, the simulation frequency is $10 \mathrm{kHz}$ and the data time window is $t_{w}=3 \mathrm{~ms}$. Since the morphological calculations are all addition and subtraction operations, the calculation time of the processor is negligible. Taking the longest timeconsuming case as an example, when a fault occurs at $F_{3}$, the propagation time of the fault traveling wave from fault point to MMC 3 is $t_{p}=0.7 \mathrm{~ms}$. In addition, based on the propagation speed of light in optical fiber being $200000 \mathrm{~km} / \mathrm{s}$, the optical fiber communication time between MMC4 and MMC 3 is $t_{c}=1.05 \mathrm{~ms}$. The total time delay is about $t_{R}=t_{p}+t_{w}+t_{c}=0.7+3.0+1.05=$ $4.75 \mathrm{~ms}$, less than $5 \mathrm{~ms}$. Therefore, the protection scheme proposed in this paper can be used as a fast backup protection scheme for the line protection of MMC HVDC grids.

\section{F. Comparison with Existing Approaches}

To compare the performance of the proposed scheme with existing approaches [11]-[13], the endurance to fault resistances, noise interference, the robustness to communication failure problems and the response time are investigated. To be fair, the approaches in [11]-[13] are all tested in the same 
This paper is a post-print of a paper submitted to and accepted for publication in IEEE Journal of Emerging and Selected Topics in Power Electronics and is subject to Institution of Electrical and Electronic Engineering Copyright. The copy of record is available at IEEE Xplore Digital Library

simulation system.

1) Comparison with reference [11]

In reference [11], a transient current correlation based pilot protection method is proposed to identify internal faults. Applying internal faults with different resistances at the middle of OHL34, the simulation results are depicted in Fig. 26. Where $I_{34 p}$ and $I_{43 p}$ are the positive pole currents at the terminals of line 34. As can be seen, when the fault resistance exceeds $100 \Omega$, the correlation coefficient is less than $0.05[11]$, indicating that the method in [11] cannot identify medium and high-resistance faults.

According to reference [11], the scheme can operate correctly under $20 \mathrm{~dB}$ Gaussian noise, but it cannot operate under $10 \mathrm{~dB}$ Gaussian noise. Moreover, the maximum tolerable synchronization error is $0.3 \mathrm{~ms}$. And the other communication problems, such as data error and data loss have not been studied in [11].

The duration of the data window in [11] is $0.5 \mathrm{~ms}$, i.e., $t_{w}=0.5 \mathrm{~ms}$. Thus, the response time is $t_{R}=t_{p}+t_{w}+t_{c}=0.7+0.5+1.05=$ $2.25 \mathrm{~ms}$.
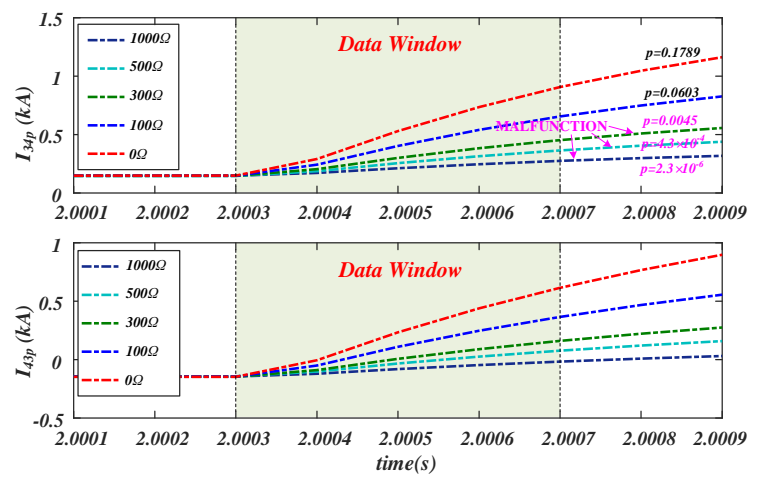

Fig. 26 Simulation results of the approach proposed in reference [11].

2) Comparison with reference [12]

Reference [12] proposed a pilot protection scheme based on comparing the correlation coefficient $C$ of the traveling waves at both ends. The larger the $C$, the smaller difference between current data at both ends. The threshold of $C$ is 0.75 .

According to reference [12], the scheme can correctly operate under a fault with $1000 \Omega$ resistance. To test the endurance to different noise levels, the faults considering different noise are applied at $F_{2}$ and $F_{5}$. Fig. 27 shows the simulation results. As can be seen, the external faults at $F_{2}$ with $500 \Omega$ and $1000 \Omega$ resistances will be misidentified as internal faults under the noise of $10 \mathrm{~dB}$. Moreover, the efficiency under $0.5 \mathrm{~ms}$ synchronization error is verified. However, the performance under data error and data loss has not been studied.

The duration of data window $t_{w}$ is $2 \mathrm{~ms}$ in [12]. Thus, the response time of the method in the reference [12] is $t_{R}=t_{p}+t_{w}+t_{c}=$ $0.7+2+1.05=3.75 \mathrm{~ms}$.

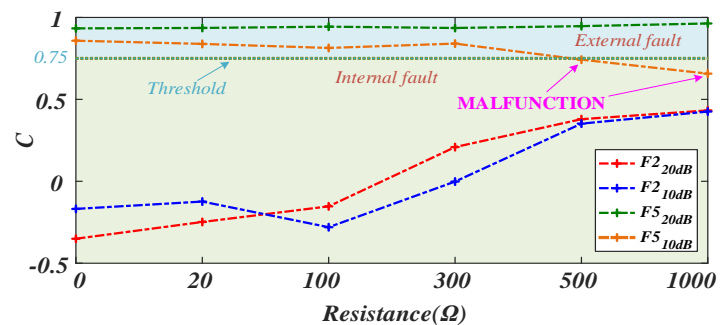

Fig. 27 Simulation results in reference [12] considering different noise interference.

3) Comparison with reference [13]

Reference [13] proposed a pilot protection scheme based on MAF(Moving Average Filter) processed DC power difference. If the calculated DC power difference $P_{M N}$ (or $P_{N M}$ ) is not equal to 0 , it means that there is an internal fault. The detailed protection criterion is shown as:

$$
\left\{\begin{array}{l}
P_{M N_{-} M A F}<-P_{s e t} \& P_{N M_{-} M A F}>P_{s e t}, P-P T G \\
P_{M N_{-} M A F}>P_{s e t} \& P_{N M_{-} M A F}<-P_{s e t}, N-P T G \\
P_{M N_{-} M A F}>P_{s e t} \& P_{N M_{-} M A F}>P_{s e t}, P T P
\end{array}\right.
$$

In equation (18), the threshold $P_{\text {set }}$ is set to be 0.1 times of the DC transmission power. The data with the subscript ' $M A F$ ' represents that the data has been processed by $M A F$.

According to reference [13], the scheme can operate correctly under internal faults with $500 \Omega$ resistance. However, the identification of external faults has not been studied in [13]. To study the ability of external faults identification, external PTP faults with different resistances are applied at $F_{5}$. Since the transmission power of OHL 34 is about $3000 \mathrm{MW}$, the $P_{\text {set }}$ can be selected as 300MW. The simulation results are shown in Fig. 28. As can be seen, the scheme will malfunction under an external fault with a small fault resistance. In Fig. 28, $P_{M N_{-} M A F}$ and $P_{N M \_M A F}$ are both larger than $P_{\text {set }}$, which means that it is wrongly identified as an internal PTP fault.

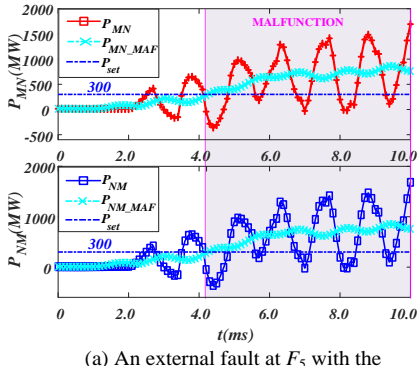

(a) An external fault at $F_{5}$ with the resistance of $0 \Omega$

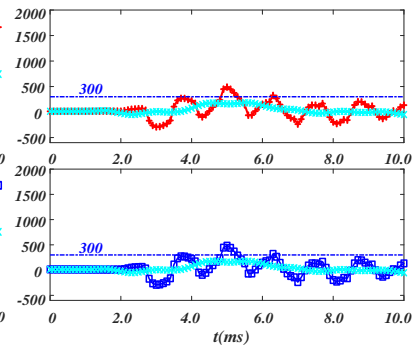

(b) An external fault at $F_{5}$ with the resistance of $500 \Omega$
Fig. 28 The performance of the scheme proposed in reference[13] under external faults with different resistances.

To study the robustness to noise interference, an internal PPTG fault considering $10 \mathrm{~dB}$ noise is applied at $F_{2}$. The simulation results are shown in Fig. 29. It can be seen that the scheme operates correctly under $10 \mathrm{~dB}$ noise. However, the effect of communication problems has not been studied.

In reference [13], the duration of data window is about $10 \mathrm{~ms}$. Thus, the response time of the method is $t_{R}=t_{p}+t_{w}+t_{c}=$ $0.7+10+1.05=11.75 \mathrm{~ms}$. 
This paper is a post-print of a paper submitted to and accepted for publication in IEEE Journal of Emerging and Selected Topics in Power Electronics and is subject to Institution of Electrical and Electronic Engineering Copyright. The copy of record is available at IEEE Xplore Digital Library
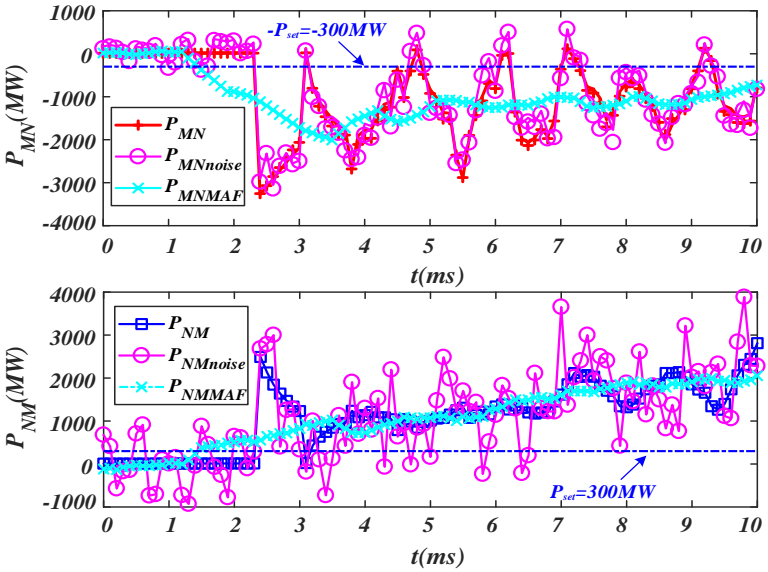

Fig. 29 Simulation results of an internal P-PTG fault at F2 considering 10dB noise.

Table 6 Comparison between Ref. [11]-[13] and the proposed method.

\begin{tabular}{|c|c|c|c|c|c|}
\hline \multicolumn{2}{|c|}{ Performance } & $\begin{array}{l}\text { Ref } \\
{[11]}\end{array}$ & $\begin{array}{l}\text { Ref } \\
{[12]}\end{array}$ & $\begin{array}{l}\text { Ref } \\
{[13]}\end{array}$ & $\begin{array}{c}\text { Proposed } \\
\text { method }\end{array}$ \\
\hline \multirow{2}{*}{$\begin{array}{l}\text { Capability to } \\
\text { identify } \\
\text { different faults }\end{array}$} & $\begin{array}{c}\text { Max fault } \\
\text { resistance }(\Omega)\end{array}$ & 100 & 1000 & 500 & 1000 \\
\hline & Max noise $(\mathrm{dB})$ & 20 & 20 & 10 & 10 \\
\hline \multirow{3}{*}{$\begin{array}{l}\text { Robustness to } \\
\text { communication } \\
\text { problems }\end{array}$} & $\begin{array}{c}\text { Max } \\
\text { synchronization } \\
\text { error }(\mathrm{ms})\end{array}$ & 0.3 & 0.5 & l & No limit \\
\hline & $\begin{array}{c}\text { Max data error } \\
(\%)\end{array}$ & I & I & I & $1 \%$ \\
\hline & $\begin{array}{c}\text { Max data loss } \\
(\%)\end{array}$ & I & / & I & $5 \%$ \\
\hline \multicolumn{2}{|c|}{ Response time (ms) } & 2.25 & 3.75 & 11.75 & 5 \\
\hline
\end{tabular}

In conclusion, the overall comparison between references [11]-[13] and the proposed method in this paper are summarized in

. It can be seen that the proposed scheme has the best performance in terms of capability to identify different faults. But the response time of the proposed scheme is not the fastest among those methods. However, since the primary protection of MMC HVDC grid is required to realize correct fault identification within 3 5ms[31][32], the proposed scheme is still suitable as quick backup protection for MMC-HVDC grids.

\section{CONCLUSION}

This paper proposes a novel differential pilot protection scheme that can withstand different communication problems. This scheme uses MMG to locate the fault traveling wave arrival time and uses a non-linear filtering algorithm (morphological filtering) to pre-process the communicated samples. A large number of simulation results show that the scheme has the ability to withstand severe data errors and a certain degree of data loss. Meanwhile, the proposed scheme avoids the influence of synchronization errors between the clock of both stations without using any methods to correct time error.

In this specific four-terminal meshed grid topology, the maximum fault resistance is $1000 \Omega$. Using the line-mode component of the fault voltage traveling wave as the communication data can comprehensively identify the fault types at low communication burden cost. The protection scheme is resilient to severe communication failures of $1 \%$ bit error rate and $5 \%$ data loss with the longest time delay of fault detection less than $5 \mathrm{~ms}$. This protection scheme can well serve as back-up protection for the MMC HVDC grids and be applied in other grid topologies.

\section{REFERENCES}

[1] W. Xiang, S. Yang and J. Wen, "ANN-based robust DC fault protection algorithm for MMC high-voltage direct current grids," IET Renew. Power Gener., vol. 14, no. 2, pp. 199-210, Feb. 2020.

[2] X. Lu, W. Xiang, W. Lin, J. Wen, "Small-signal modeling of MMC based DC grid and analysis of the impact of DC reactors on the small-signal stability," Int. J. Electr. Power Energy Syst., vol. 101, pp. 25-37, Oct. 2018.

[3] Y. Wang, C. Wang, L. Xu, et al, "Adjustable Inertial Response from the Converter with Adaptive Droop Control in DC Grids," IEEE Trans. Smart Grid, vol. 10, no. 3, pp. 3198-3209, May 2019.

[4] D. Jovcic, M. Taherbaneh, J. Taisne, et al, "Offshore DC Grids as an Interconnection of Radial Systems: Protection and Control Aspects," IEEE Trans. Smart Grid, vol. 6, no. 2, pp. 903-910, March 2015.

[5] W. Xiang, S. Yang, L. Xu, et al, "A Transient Voltage-Based DC Fault Line Protection Scheme for MMC-Based DC Grid Embedding DC Breakers," IEEE Trans. Power Del., vol. 34, no. 1, pp. 334-345, Feb. 2019.

[6] O. Cwikowski, H. R. Wickramasinghe, G. Konstantinou, et al, "Modular Multilevel Converter DC Fault Protection," IEEE Trans. Power Del., vol. 33, no. 1, pp. 291-300, Feb. 2018.

[7] S. Yang, W. Xiang, R. Li, et al, "An Improved DC fault Protection Algorithm for MMC HVDC Grids based on Modal Domain Analysis," IEEE J. Emerg. Sel. Top. Power Electron, early access, to be published.

[8] Q. Huang, G. Zou, S. Zhang, et al, "A Pilot Protection Scheme of DC Lines for Multi-Terminal HVDC Grid," IEEE Trans. Power Del., vol. 34, no. 5, pp. 1957-1966, Oct. 2019.

[9] M. J. Hossain, S. P. Majumder, "Bit Error Rate (BER) performance analysis of an optical fiber communication system with a Multi-core fiber (MCF)," 2015 International Conf. on Electrical \& Electronic Engineering (ICEEE), Rajshahi, 2015, pp. 173-176.

[10] Y. Xue, M. Ni, W. Yu, "Approach for studying the impact of communication failures on power grid," 2016 IEEE Power and Energy Society General Meeting (PESGM), Boston, MA, 2016, pp. 1-5.

[11] K. Jia, C. Wang, T. Bi, et al, "Transient Current Correlation based Protection for DC Distribution System," IEEE Trans. Ind. Electron., vol. 67, no. 11, pp. 9927-9936, Nov. 2020.

[12] Y. Wang, Z. Hao, B. Zhang, et al, "A Pilot Protection Scheme for Transmission Lines in VSC-HVDC Grid Based on Similarity Measure of Traveling Waves," IEEE Access, vol. 7, pp. 7147-7158, Jan. 2019.

[13] L. Ning, X. Zheng, N. Tai, et al, "A novel pilot protection scheme for MMC-HVDC transmission lines," 2017 IEEE Energy Conversion Congress and Exposition (ECCE), Cincinnati, OH, 2017, pp. 105-110.

[14] N. Jin, X. Lin, Jia. Xing, et al. "Research on Multi-terminal Current Differential Protection Criterion With High Sensitivity and Synchronization Error Tolerance Capability," IEEE Trans Power Del, vol. 33, no. 6, pp. 3085-3094, Dec. 2018.

[15] M. Shin, M. Park, D. Oh, et al. "Survey on the Clock Synchronization Schemes for Propagation Delay Measurement," Int. J. Advanced Sci. and Techno., vol. 35, pp.139-149, Oct. 2011.

[16] B. Kasztenny, N. Fischer, B. Le. "Fallback algorithms for line current differential protection applied with asymmetrical channels upon the loss of time reference," 11th IET International Conf. on Developments in Power Syst. Protection (DPSP 2012), Birmingham, UK, 2012, pp. 1-6.

[17] W. Wang, X. Liu, H. Yu, et al, "Application of Optical Fiber Differential Protection in High Voltage Power Network," 2017 4th International Conf. on Information Science and Control Engineering (ICISCE), Changsha, 2017, pp. 1422-1424.

[18] T. Cao, C. Dai, J. Chen and Z. Yu, "A new method of channel monitoring for fiber optic line differential protection," 2008 China International Conf. on Electricity Distribution, Guangzhou, 2008, pp. 1-4.

[19] C. Zhang, G. Song and T. Wang, "Analysis of traveling wave front and its application in fault location," 8th Renew. Power Gener. Conf. (RPG 2019), Shanghai, China, 2019, pp. 1-6.

[20] M. Xu, Z. Cai, X. Li, et al, "Analysis of Fault on HVDC Transmission System Considering Frequency-dependent Parameters and HVDC 
This paper is a post-print of a paper submitted to and accepted for publication in IEEE Journal of Emerging and Selected Topics in Power Electronics and is subject to Institution of Electrical and Electronic Engineering Copyright. The copy of record is available at IEEE Xplore

Digital Library

control," Automation of Electric Power Syst., vol. 39, no. 11, pp 37-44, Jun. 2015.

[21] F. Kong, Z. Hao, S. Zhang, et al, "Development of a Novel Protection Device for Bipolar HVDC Transmission Lines," IEEE Trans. Power Del., vol. 29, no. 5, pp. 2270-2278, Oct. 2014.

[22] Q. Wu, Z. Lu, T. Ji, "Protective relaying of power systems using mathematical morphology," Berlin: Springer, 2009, pp, 15-25.

[23] X. Lin, L. Zou, Q. Tian, et al., "A series multiresolution morphological gradient-based criterion to identify CT saturation," IEEE Trans. Power Del., vol. 21, no. 3, pp. 1169-1175, Jul. 2006.

[24] S. Jamali, S. S. Mirhosseini, "Protection of transmission lines in multiterminal HVDC grids using travelling waves morphological gradient," Int. J. Electr. Power Energy Syst., vol. 108, no. 13, pp 125-134, Jun 2019.

[25] H. M. M. Maruf, F. Kamal and B. H. Chowdhury, "Mathematical Morphological Filter Based Protection Scheme for DC Ring Microgrid System," 2018 North American Power Symposium (NAPS), Fargo, ND, 2018, pp. 1-6.

[26] A. Gartia, A. Gartia, A. Gulati, et al, "Microcontroller based line differential protection using fiber optic communication," 2013 IEEE Innovative Smart Grid Technologies-Asia (ISGT Asia), Bangalore, 2013, pp. 1-4.

[27] W. Dang, "Implement of HDB3 coder in base-band system," $20122^{\text {nd }}$ International Conf. on Consumer Electronics, Communications and Networks (CECNet), Yichang, 2012, pp. 3273-3274.

[28] P. D. S. Assala, H. Chen and T. Ji, "Power system overvoltage detection based on a morphological filtering algorithm,"2013 IEEE Power \& Energy Society General Meeting, Vancouver, BC, 2013, pp. 1-4.

[29] L. Zou, Q. Zhao, X. Lin, et al., "Improved phase selector for unbalanced faults during power swings using morphological technique," IEEE Trans. Power Del., vol. 21, no. 4, pp. 1847-1855, Oct. 2006.

[30] Design technical rules for transmission of protection information on radio link system, DL/T 5062-1996, 1996.

[31] L. Tang, X. Dong, B. Wang, S. Shi, Q. Dong and T. Coombs, "Protection Configuration and Scheme for the Transmission Line of VSC-HVDC Grid," 2018 IEEE Power \& Energy Society General Meeting (PESGM), Portland, OR, 2018, pp. 1-5.

[32] G. Liu, F. Xu, Z. Xu, Z. Zhang and G. Tang, "Assembly HVDC Breaker for HVDC Grids with Modular Multilevel Converters," IEEE Trans. Power Electron., vol. 32, no. 2, pp. 931-941, Feb. 2017.

\section{BIOGRAPHIES}

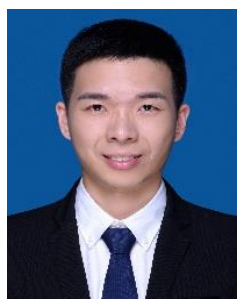

Wang Xiang (S'16-M'17) received his B.Eng. and $\mathrm{PhD}$ degrees both in electrical engineering from Huazhong University of Science and Technology (HUST), China in 2012 and 2017, respectively. He was a visiting student at the University of Aberdeen and the University of Strathclyde in 2014 and 2016 respectively. Currently, he is a research associate with the University of Strathclyde since 2018. His main research interests include MMCHVDC, high power dc/dc converters and dc grids.

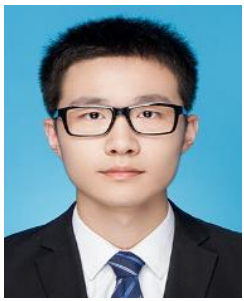

Haobo Zhang obtained his B.E. degree in electrical engineering from Huazhong University of Science and Technology (HUST), China, in 2020. He is currently pursuing his master degree at HUST. His research interests include dc fault protection of MMC-HVDC and dc grids.

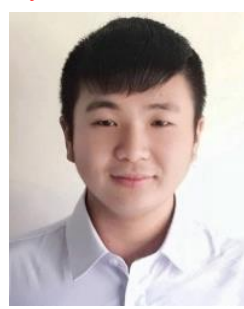

Saizhao Yang obtained his B.E. degree in electrical engineering from Huazhong University of Science and Technology (HUST), China, in 2018. He is currently pursuing his $\mathrm{PhD}$ degree at HUST. His research interests include dc fault protection of MMC-HVDC and dc grids.

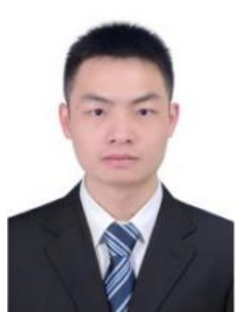

Meng Zhou was born in Hunan, China, in 1987. He received the $\mathrm{Ph}$.D. degree in electrical engineering from the School of Electrical and Electronic Engineering, Huazhong University of Science and Technology, Hubei, China, in 2019. He is currently a Postdoctoral Researcher with the School of Electrical and Electronic Engineering, Huazhong University of Science and Technology. His current research interests include voltage-source converter-HVdc and dc circuit breaker.

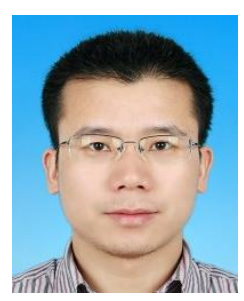

Weixing Lin (M'13) obtained his B.E. and PhD degrees in electrical engineering from Huazhong University of Science and Technology (HUST), China, in 2008 and 2014 respectively. He was a research fellow at University of Aberdeen during 2012-2016. He is currently the chief engineer of HVDC division at TBEA China Xinjiang Sunoasis Co. Ltd. His research interests are HVDC, MMC, dc-dc autotransformer, DC grids.

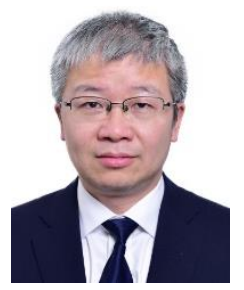

Jinyu Wen (M'10) received his B.Eng. and Ph.D. degrees all in electrical engineering from Huazhong University of Science and Technology (HUST), Wuhan, China, in 1992 and 1998, respectively. He was a visiting student from 1996 to 1997 and a research fellow from 2002 to 2003 all at the University of Liverpool, UK, and a senior visiting researcher at the University of Texas at Arlington, USA in 2010. From 1998 to 2002 he was a director engineer in XJ Electric Co. Ltd. in China. In 2003 he joined the HUST and now is a Professor at HUST. His current research interests include renewable energy integration, energy storage application, DC grid, and power system operation and control. 